\title{
A probabilistic approach for cross-spectral matrix denoising: Benchmarking with some recent methods
}

\author{
Alice Dinsenmeyer ${ }^{1,2}$, Jérôme Antoni $^{1}$, Quentin Leclère ${ }^{1}$, and Antonio Pereira ${ }^{2}$ \\ ${ }^{1}$ Univ Lyon, INSA Lyon, Laboratoire Vibrations Acoustique, F-69621 Villeurbanne, France \\ ${ }^{2}$ Univ Lyon, École Centrale de Lyon, INSA Lyon, Université Claude Bernard Lyon I, CNRS, Laboratoire de Mécanique des \\ Fluides et d'Acoustique, UMR 5509, F-69134 Écully, France
}

May 2020

\begin{abstract}
Array measurements can be contaminated by strong noise, especially when dealing with microphones located near or in a flow. The denoising of these measurements is crucial to allow efficient data analysis or source imaging. In this paper, a denoising approach based on a Probabilistic Factor Analysis (PFA) is proposed. It relies on a decomposition of the measured cross-spectral matrix (CSM) using the inherent correlation structure of the acoustical field and of the flow-induced noise. This method is compared with three existing approaches, aiming at denoising the CSM, without any reference or background noise measurements and without any information about the sources of interest. All these methods make the assumption that the noise is statistically uncorrelated over the microphones, and only one of them significantly impairs the off-diagonal terms of the CSM. The main features of each method are first reviewed, and their performances are then evaluated by way of numerical simulations along with measurements in a closed-section wind tunnel.
\end{abstract}

\section{Introduction}

Nowadays, the use of multichannel measurements is a current practice for the experimental characterization of noise sources. Automotive, health-care and aeronautics are a few examples of industries where array systems are largely employed. In this context, several techniques based on advanced signal processing have been developed. Some of them are in the field of acoustic imaging techniques, where the main goals are to locate, quantify and rank noise sources of different origins. Common to all experimental approaches, is the presence of (extraneous) measurement noise affecting the quality of measured signals. Examples of measurement noise are for instance, electronic noise, ambient noise or flow-induced noise, among others.

The issue of measurement noise has been investigated since the late $70 \mathrm{~s}$ in the signal processing community. One classical work is the well-known multiple signal classification (MUSIC) algorithm [1]. The MUSIC algorithm exploits the eigenstructure of an estimated covariance matrix in order to distinguish signal from noise. The algorithm is based on the assumption that signal and noise span different subspaces, which can be identified by a clear transition if one looks at the eigenvalue spectrum of the covariance matrix. However, this observation holds only for high signal-to-noise ratio (SNR) scenarios and small number (as compared to the number of microphones) of uncorrelated sources. Apart from these particular cases, the eigenvalues of the covariance matrix exhibit a continuous decrease and the separation of noise and signal subspaces is more cumbersome. Also in the late 70s, Chung[2] proposed a method for the extraction of flow-induced noise from simultaneous measurements of pressure fluctuations. The method, which has then been coined as the "three-microphone method" is based on the coherence function between each microphone pair of a three-probe system. The main assumption of this approach is that the flow noise at three spatially separated transducers is uncorrelated.

In the early 90s, in the underwater acoustics community, researchers have started to deal with the scenario of poor signal-to-noise ratios [3]. In this context, a first proposition has been formulated for the particular case of uniform linear arrays. It is known that for uncorrelated sources the cross-spectral matrix (CSM) measured by this particular array under a far-field assumption has a block Toeplitz structure. Thus, denoising is performed by forcing the estimated CSM to be Toeplitz, by averaging its elements along its diagonals. In the signal processing community this method is also known as Cadzow denoising [4]. Forster and Asté [5, 6] have later generalized this technique for the case of arbitrary array configurations. The main idea therein is to first generate a subspace of Hermitian matrices based upon a model which depends on the array geometry and on the 
assumption of uncorrelated sources. Denoising is then simply performed by projecting the estimated CSM onto the designed subspace.

Aeroacoustics is another field of application that has shown interest in the problem of denoising acoustical pressure signals. This is mainly due to the fact that the signals of interest are often highly disturbed by the presence of flow-induced noise or installation effects in aerodynamic wind-tunnels for instance [7]. A widespread practice is to set the diagonal entries of an estimated cross-spectral matrix to zero. The justification behind it is that flow induced noise has generally a spatial correlation which is smaller than a typical microphone inter-spacing encountered in arrays. More advanced methods have been recently proposed in this community. For instance, Bulté[8] has applied similar ideas to the subspace approaches mentioned in the previous paragraph for the suppression of extraneous noise inherent to wind tunnel measurements. It is proposed that the noise subspace may be generated either performing a singular value decomposition of a measured "background" CSM or by using a Spherical Harmonics Decomposition of the sound field generated by multiple equivalent sources around the model. Transforming the spatially sampled pressure field to other representation spaces such as the wavenumber domain $[9,10,11]$ has recently seen much use for the separation of pressure fluctuations stemming from different origins. Ehrenfried et al. $[12,13]$ have proposed an algorithm so called BiClean which uses a wavenumber decomposition to reduce background noise from wind-tunnel array measurements.

In practical situations it is sometimes possible to measure separately the contaminating background noise, by either simply "switching off" the source of interest or without the mock-up in wind tunnel measurements for instance. This background measurement can be used to advantage to remove its influence when the total noise field is actually measured. It can be done by either simply using spectral subtraction approaches or more advanced methods that take this prior information into account in the processing, such as the work in Refs. 14, 15, 16. Removing unwanted flow-induced noise by physically suppressing it has also been proposed in the literature. It has been shown that this can be obtained by setting-up the microphones behind a Kevlar screen [17] or using a porous layer in order to absorb sound waves reaching the array at oblique incidence [18]. Another alternative might be the use of a vibrating structure (e.g. an thin plate) equipped with accelerometers [19]. It has been shown that the structure acts as a low-pass filter in the wavenumber domain, thus filtering out components with high wavenumbers associated to flow-induced noise. It is necessary though to convert the acceleration signals into wall-pressure signals using inverse techniques[20].

Speech signal processing is another field in which much research effort has been devoted to developing noise reduction methods [21]. Spectral subtraction [22] methods and subspace approaches [23] are examples of techniques proposed in this community.

The problem addressed in this paper is the suppression of uncorrelated extraneous noise over a microphone array in a blind manner. The term "blind" is to be understood as "without any measurement of a reference background noise". Indeed, it is common in practice that the separate measurement of the unwanted noise (i.e. without the source of interest) is not available, since it is the source itself that generates the extraneous noise (see the example of fan noise using in-duct measurements [24]). Moreover, no assumption is made about the source location or propagation unlike the beamforming-based methods proposed by Sijtsma et $a l .[25]$.

In the present work, a new method is proposed which is based on a Probabilistic Factor Analysis problem and three other denoising approaches from the literature are also investigated. After a section dedicated to the problem statement, an overview of all these methods is given in Sec. 3. The denoising performance of each approach is then compared first on numerical simulations (in Sec. 4) and then on real wind-tunnel measurements (in Sec. 5).

Since the aforementioned Chung's method is still widely used, it is presented in light of the other denoising methods in Appendix A, but because of its limitations, this method is not compared with the other methods for the numerical and experimental applications.

\section{Problem statement}

Let $p(\boldsymbol{r}, \omega)$ be the acoustic signal (i.e. pressure fluctuations) measured by a receiver at position $r \in \mathbb{R}^{3}$ in space at a given angular frequency $\omega$. The measured signal can be modeled as a linear combination of signals emitted by noise sources having any distribution in space. After proper discretization of the domain of interest, the signal measured at a microphone position $i$ may be simply written as:

$$
p\left(\boldsymbol{r}_{i}, \omega\right)=a\left(\boldsymbol{r}_{i}, \omega\right)+n\left(\boldsymbol{r}_{i}, \omega\right),
$$

where $n\left(\boldsymbol{r}_{i}, \omega\right)$ represents additive noise statistically independent of the signal of interest $a\left(\boldsymbol{r}_{i}, \omega\right)$. Considering that the acoustical field is spatially sampled by $M$ receiver positions, Eq. (1) may be conveniently expressed in matrix-form as

$$
p=a+n,
$$


and the acoustical field is

$$
\boldsymbol{a}=\boldsymbol{H q}
$$

where $\boldsymbol{H} \in \mathbb{C}^{M \times K}$ is a matrix that maps the acoustic pressure to K coefficients $\boldsymbol{q}$ of a given representation basis.

Let us now define the covariance matrix of measurements (i.e. the theoretical cross-spectral matrix if the covariance between Fourier coefficients is of concern) as $\boldsymbol{S}_{p p} \triangleq \mathbb{E}\left\{\boldsymbol{p} \boldsymbol{p}^{H}\right\}$, where ${ }^{H}$ stands for the conjugate transpose (Hermitian) operator and $\mathbb{E}\{\cdot\}$ is to be understood as the expected value over realizations of the stochastic process.

Therefore, from (2) and (3), the theoretical model of the cross-spectral matrix used in this work is

$$
\boldsymbol{S}_{p p}=\boldsymbol{H} \boldsymbol{S}_{q q} \boldsymbol{H}^{H}+\boldsymbol{S}_{n n} .
$$

For convenience, let us define the first term in the right hand side of Eq. (4), thereafter referred to as signal CSM, $\boldsymbol{S}_{a a}=\boldsymbol{H} \boldsymbol{S}_{q q} \boldsymbol{H}^{H}$. The theoretical CSM is thus a sum of the signal CSM related to the source of interest and the noise CSM, which gives:

$$
\boldsymbol{S}_{p p}=\boldsymbol{S}_{a a}+\boldsymbol{S}_{n n},
$$

In practice, whenever the pressure field may be assumed statistically stationary (i.e. ergodic stochastic process), an estimate of the theoretical CSM may be obtained from averaging the measurements over a finite number of snapshots:

$$
\hat{\boldsymbol{S}}_{p p}=\frac{1}{N_{s}} \sum_{j=1}^{N_{s}} \boldsymbol{p}_{j} \boldsymbol{p}_{j}^{H},
$$

where the subscript $j$ refers to the $j^{\text {th }}$ snapshot, $N_{s}$ is the number of snapshots, and the superscript $\hat{\imath}$ is used throughout the paper to define an estimated quantity.

Using this estimate, when summing the acoustical field and the noise as in (4), averages of cross terms such as $(\boldsymbol{H q}) \boldsymbol{n}^{H}$ are supposed to be negligible, which is not necessarily the case in practice if the number of snapshots is low.

Finally, the denoising problem of interest in this paper is the following:

Problem 1 Given an estimate of the CSM $\hat{\boldsymbol{S}}_{p p} \approx \boldsymbol{S}_{a a}+$ $\boldsymbol{S}_{n n}$, where $\boldsymbol{S}_{a a}$ and $\boldsymbol{S}_{n n}$ are both unknown, recover $\boldsymbol{S}_{a a}$.

It is noteworthy that the noise power is allowed to vary between different microphones and to be much higher than the acoustic source power.

In order to solve this problem, it is possible to exploit the difference of structure of the two terms $\boldsymbol{S}_{a a}$ and $\boldsymbol{S}_{n n}$. First, the spatial coherence of the noise field is supposed to be smaller than the microphone inter-spacing, that is, the noise is assumed to be spatially uncorrelated over the microphone array. Second, the sound field is correlated by the propagation effect, that is, the spatial coherence associated to the source of interest is much greater than the noise spatial coherence.

\section{Methods to reduce strong uncorrelated noise from multi- channel measurements}

\subsection{Diagonal reconstruction}

As discussed in Section 2, it is assumed that the noise correlation length is smaller than the microphone interspacing. Following this assumption, the theoretical noise CSM is a diagonal matrix. From Eq. (5), the measured CSM can then be written as:

$$
\hat{\boldsymbol{S}}_{p p} \approx \boldsymbol{S}_{a a}+\left\lceil\boldsymbol{\sigma}^{2}\right\rfloor .
$$

where the notation $\left\lceil\boldsymbol{\sigma}^{2}\right\rfloor$ stands for a diagonal matrix whose diagonal entries are the elements in vector $\boldsymbol{\sigma}^{2}$.

In this section, we describe three methods from the aeroacoustic literature used to reduce the self-induced noise concentrated on the diagonal of the measured CSM. These methods all propose to minimize the diagonal elements, while keeping the denoised CSM positive semi-definite, which can formulated as follows:

$$
\begin{aligned}
& \text { maximize }\left\|\boldsymbol{\sigma}^{2}\right\|_{1} \\
& \text { subject to } \hat{\boldsymbol{S}}_{p p}-\left\lceil\boldsymbol{\sigma}^{2}\right\rfloor \geq 0
\end{aligned}
$$

where $\|\cdot\|_{1}$ is the $\ell_{1}$ norm. Each method solves this problem in a different way.

\subsubsection{Convex optimization}

Hald [26] directly uses semidefinite programming to solve this problem, more specifically the SDPT3 solver from CVX Matlab toolbox [27, 28]. This solver is an interior-point algorithm suitable for such conic optimization problems [29].

\subsubsection{Linear optimization}

Dougherty [30] restates the problem of Eq. (8) as the following linear programming problem, solved iteratively:

$$
\begin{aligned}
& \operatorname{maximize}\left\|\boldsymbol{\sigma}_{(k)}^{2}\right\|_{1} \\
& \text { subject to } \boldsymbol{V}_{(k-1)}^{H}\left(\hat{\boldsymbol{S}}_{p p}-\left\lceil\boldsymbol{\sigma}^{2}\right\rfloor_{(k)}\right) \boldsymbol{V}_{(k-1)} \geq 0
\end{aligned}
$$

at the $k^{\text {th }}$ iteration. $\boldsymbol{V}_{(k-1)}$ are the eigenvectors of $\hat{\boldsymbol{S}}_{p p}-$ $\left\lceil\boldsymbol{\sigma}^{2}\right\rfloor_{(1, \ldots, k-1)}$, concatenated from the $k-1$ previous 
iterations. This problem is later solved using the dualsimplex algorithm from the Matlab linprog function.

The concatenation of the eigenvectors increases the problem dimension - and therefore the calculation time - at each iteration. Consequently, the convergence can be very slow and the final denoised CSM may not be semi-positive definite.

\subsubsection{Alternating projections}

The minimization problem given by Eq. (8) can also be solved by an Alternating Projections algorithm, as proposed in Ref. 31. In this case,Alternating Projections aims at finding the intersection between 2 convex sets that are the positive semi-definite matrices (i.e. nonnegativity of eigenvalues) and the matrices with the same extra-diagonal elements as the measured CSM. For the sake of clarity, Algorithm 1 is the pseudo-code of this procedure.

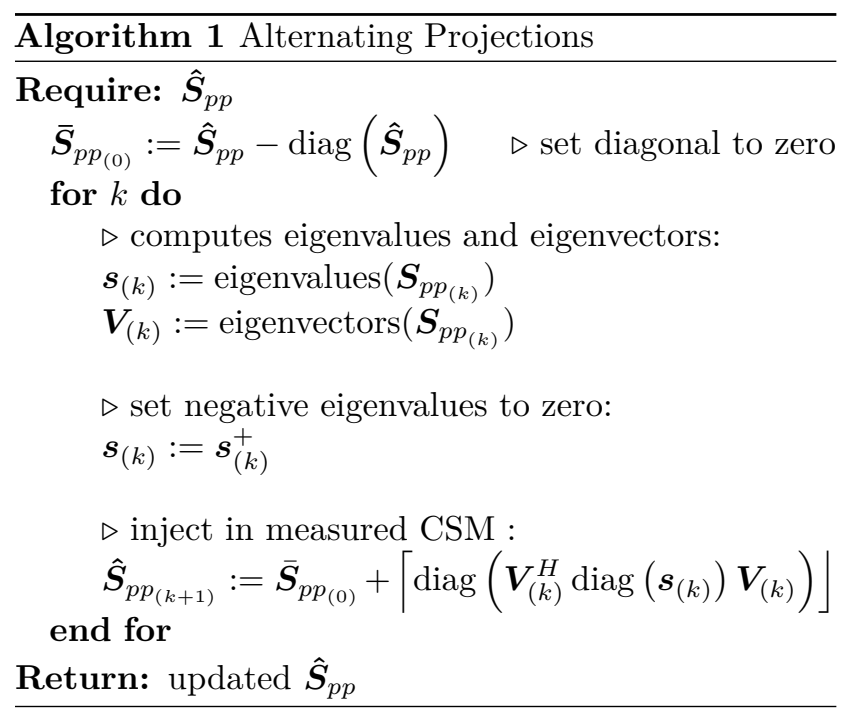

\subsection{Robust Principal Component Anal- ysis}

Another strategy to solve the Problem 1 is to use two particular properties of $\boldsymbol{S}_{a a}$ and $\boldsymbol{S}_{n n}$, namely, lowrankness and sparsity.

Let us start with the discussion on the physical interpretation of the rank of a covariance matrix in acoustic signal processing. It has been shown elsewhere that the rank is directly related to the number of statistically independent noise sources $[32,33]$ at the origin of a given acoustical field. In other words, the rank gives the number of uncorrelated components that are necessary in order to reproduce a particular sound field. From an experimental point of view, the number of acquisition channels in recent systems has been rapidly increasing. It is common nowadays to take measurements with a large number of simultaneously acquired signals, leading to high-dimensional data. Thus, in many practical situations, the number of independent sources of interest is much lower than the number of measurement channels. This justifies the assumption of a low-rank model to cross-spectral matrices in acoustics.

The use of a sparse model for a CSM of uncorrelated noise has been discussed in Section 2: the CSM tends to be diagonal when the number of snapshots tends to infinity. Thus, when the number of channels is large, the noise CSM may be approximated by a sparse matrix, since the number of non-zero diagonal elements is much less than the null off-diagonal ones.

Finally, the decomposition of the CSM in sparse and low-rank matrices can be written as the following optimization problem:

$$
\begin{aligned}
& \text { minimize }\left\|\hat{\boldsymbol{S}}_{a a}\right\|_{*}+\lambda\left\|\hat{\boldsymbol{S}}_{n n}\right\|_{1} \\
& \text { subject to } \hat{\boldsymbol{S}}_{a a}+\hat{\boldsymbol{S}}_{n n}=\hat{\boldsymbol{S}}_{p p}
\end{aligned}
$$

The nuclear norm $\|\cdot\|_{*}$ (sum of the eigenvalues) and the $\ell_{1}$ norm $\left(\|\boldsymbol{A}\|_{1}=\sum_{i j}\left|\boldsymbol{A}_{i j}\right|\right)$ are convex relaxations of low rank and sparsity constraints, respectively. The trade-off between sparsity of the noise and low-rankness of the source CSM is handled by the regularization parameter $\lambda$. The regularization strategies are multiple and not detailed here. Denoising results are presented below for two regularization parameters: $\lambda_{o p t}$ which gives the smallest error (known only for simulations) and the constant parameter $\lambda=M^{-\frac{1}{2}}$ proposed in Ref. 34.

This procedure, known as Robust Principal Component Analysis (RPCA), has been used by Finez et al. [35] and Amailland et al.[36] to denoise aeroacoustic and hydroacoustic data. A collection of algorithms is available to solve this convex problem. The reader can refer to the LRSLibrary [37, 38] in which the Accelerated Proximal Gradient algorithm, developed by Wright et al.[34], is used for the denoising applications of the present paper.

\subsection{Canonical Coherence Analysis}

The use of Canonical Coherence Analysis (CCA) to denoise the measured CSM in a context of aeroacoustic measurements has been introduced recently by Hald[39]. The principle of CCA is to find the linear combination of two subgroups of sensors with the highest mutual correlation. The vector of pressure measurements $\boldsymbol{p}_{j}$ at the $j^{\text {th }}$ snapshot is divided into two sub-sets $\boldsymbol{x}_{j}$ and $\boldsymbol{y}_{j}$ of $I$ and $J$ channels, with $M=I+J$, such that

$$
\boldsymbol{x}_{j}=\boldsymbol{L}_{x} \boldsymbol{c}_{j}+\boldsymbol{n}_{x_{j}} \text { and } \boldsymbol{y}_{j}=\boldsymbol{L}_{y} \boldsymbol{c}_{j}+\boldsymbol{n}_{y_{j}},
$$

with $\boldsymbol{c}_{j}$ a vector of $N$ uncorrelated equivalent sources, where $N \leq \min (I, J)$. Setting $\mathbb{E}\left\{\boldsymbol{c} \boldsymbol{c}^{H}\right\}=\boldsymbol{I}_{N}$ (with 
$\boldsymbol{I}_{N}$ the identity matrix of dimension $N$ ), without loss of generality since matrices $\boldsymbol{L}_{x}$ and $\boldsymbol{L}_{y}$ can always be defined accordingly, one has

$$
\begin{aligned}
& \boldsymbol{S}_{x x}=\boldsymbol{L}_{x} \boldsymbol{L}_{x}^{H}+\mathbb{E}\left\{\boldsymbol{n}_{x} \boldsymbol{n}_{x}^{H}\right\}, \\
& \boldsymbol{S}_{y y}=\boldsymbol{L}_{y} \boldsymbol{L}_{y}^{H}+\mathbb{E}\left\{\boldsymbol{n}_{y} \boldsymbol{n}_{y}^{H}\right\}, \\
& \boldsymbol{S}_{x y}=\boldsymbol{L}_{x} \boldsymbol{L}_{y}^{H} .
\end{aligned}
$$

The last equation shows that noise is canceled if it is uncorrelated between the two groups $x$ and $y$. This gives hope to get estimates of the factors $\boldsymbol{L}_{x}$ and $\boldsymbol{L}_{y}$, say $\hat{\boldsymbol{L}}_{x}$ and $\hat{\boldsymbol{L}}_{y}$, from the measured CSM $\hat{\boldsymbol{S}}_{x y}$. If so, the signal CSM can in turn be estimated as

$$
\hat{\boldsymbol{S}}_{a a}=\left(\begin{array}{c}
\hat{\boldsymbol{L}}_{x} \\
\hat{\boldsymbol{L}}_{y}
\end{array}\right)\left(\begin{array}{c}
\hat{\boldsymbol{L}}_{x} \\
\hat{\boldsymbol{L}}_{y}
\end{array}\right)^{H}
$$

Estimates of the factors are obtained from a generalized singular value decomposition (GSVD)

$$
\hat{\boldsymbol{S}}_{x y}=\tilde{\boldsymbol{U}} \boldsymbol{\Sigma} \tilde{\boldsymbol{V}}^{H},
$$

where $\tilde{\boldsymbol{U}}=\hat{\boldsymbol{S}}_{x x}^{1 / 2} \boldsymbol{U}$ and $\tilde{\boldsymbol{V}}=\hat{\boldsymbol{S}}_{y y}^{1 / 2} \boldsymbol{V}$ and with $\boldsymbol{U}$ and $\boldsymbol{V}$ the left and right singular vectors of $\hat{\boldsymbol{S}}_{x x}^{-1 / 2} \hat{\boldsymbol{S}}_{x y} \hat{\boldsymbol{S}}_{y y}^{-1 / 2}$, respectively. Upon truncating the GSVD to its $N$ leading singular values,

$$
\hat{\boldsymbol{L}}_{x}=\tilde{\boldsymbol{U}}_{N} \boldsymbol{\Sigma}_{N}^{1 / 2} \text { and } \quad \hat{\boldsymbol{L}}_{y}=\tilde{\boldsymbol{V}}_{N} \boldsymbol{\Sigma}_{N}^{1 / 2} .
$$

where $\tilde{\boldsymbol{U}}_{N}$ (resp. $\tilde{\boldsymbol{V}}_{N}$ ) stands for the matrix containing the corresponding $N$ "leading" left (resp. right) singular vectors, the complete denoised CSM reads

$$
\tilde{\boldsymbol{S}}_{a a}=\left(\begin{array}{c}
\hat{\boldsymbol{S}}_{x x}^{1 / 2} \boldsymbol{U}_{N} \boldsymbol{\Sigma}_{N}^{1 / 2} \\
\hat{\boldsymbol{S}}_{y y}^{1 / 2} \boldsymbol{V}_{N} \boldsymbol{\Sigma}_{N}^{1 / 2}
\end{array}\right)\left(\begin{array}{c}
\hat{\boldsymbol{S}}_{x x}^{1 / 2} \boldsymbol{U}_{N} \boldsymbol{\Sigma}_{N}^{1 / 2} \\
\hat{\boldsymbol{S}}_{y y}^{1 / 2} \boldsymbol{V}_{N} \boldsymbol{\Sigma}_{N}^{1 / 2}
\end{array}\right)^{H}
$$

The thresholding of the singular values proposed in Ref. 39 is empirical, and for the applications presented in Sec. 4 and 5, the same strategy is adopted, since the application is very similar.

The classical CCA is not able to extract a number of canonical components that is higher than the number of channels in the smallest subgroup, which is a limitation to represent a high number of uncorrelated sources. Therefore, Hald proposed to overcome this limitation by performing several CCA iteratively with different sub-groups, on the residuals of the denoised matrix from the previous iteration. However, the number of iterations has to be adapted to the number of sources in the acoustical field. In the following, the sub-groups and the number of iterations are chosen to be the same as the empirical recommendations given in Ref. 39. Any other sub-arrays of $\frac{M}{2}$ sensors could have been used. An idea of the procedure is given in Algorithm 2.

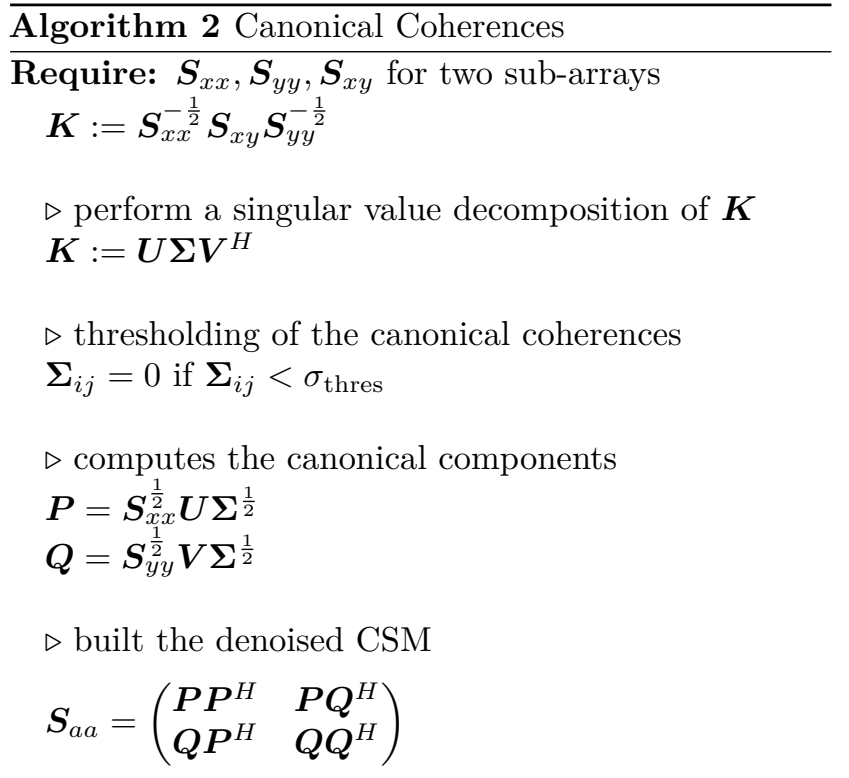

Repeat: for other sub-arrays, depending on the number of significant canonical coherences

Return: $\boldsymbol{S}_{a a}$

\subsection{Probabilistic Factor Analysis}

Probabilistic factor analysis (PFA) is an inference problem that aims at fitting the measurements with the following model:

$$
\boldsymbol{p}_{j}=\boldsymbol{L} \boldsymbol{c}_{j}+\boldsymbol{n}_{j} \quad j=1, \ldots, N_{s}
$$

where $\boldsymbol{c}$ is the vector of $\kappa<M$ equivalent sources or latent factors (the same as in Eq. (11)), $\boldsymbol{L} \in \mathbb{C}^{M \times \kappa}$ is the matrix that mixes the factors, $\boldsymbol{n}$ is a vector of residual errors (independent of the factors) and $j$ refers to the $j^{\text {th }}$ snapshot.

Similarly to Principal Component Analysis (PCA), Factor Analysis is a mean to reduce data dimension by fitting the signal CSM to a low-rank matrix model. In PCA, the data are decomposed into a limited number of independent variables whose contributions to measurement points are orthogonal. PFA also involves a linear combination of variables but with contributions to measurement points that are not necessarily orthogonal. Therefore, PFA allows the incorporation of more assumptions about the latent structures of the data.

No physical interpretation can be made from $\boldsymbol{L}$ and $\boldsymbol{c}$, since $\boldsymbol{L} \neq \boldsymbol{H}$ and $\boldsymbol{c} \neq \boldsymbol{q}$ in general. Indeed, if the $\boldsymbol{L} \boldsymbol{c}$ decomposition of the acoustical field $\boldsymbol{a}$ is unique, $\boldsymbol{L}$ and $c$ are not.

In the context of Probabilistic Factor Analysis, all the unknown parameters of the model are seen as random variables, with an assigned probability density function (PDF). In the classical PFA model, all the assigned PDFs are Gaussian. 
Throughout the paper, the term Gaussian refers in a short way to the class of circularly-symmetric multivariate complex Gaussian distributions, and is noted $\mathcal{N}_{\mathbb{C}}(\boldsymbol{\mu}, \boldsymbol{\Omega})$ where $\boldsymbol{\mu}$ is the mean vector and $\boldsymbol{\Omega}$ the covariance matrix.

Using the notation $[x]$ for the conditional PDF of $x$, the assigned PDF for the factors and the noise vector are the following:

$$
[\boldsymbol{c}]=\mathcal{N}_{\mathbb{C}}\left(0, \boldsymbol{I}_{\kappa}\right) \quad \text { and } \quad[\boldsymbol{n}]=\mathcal{N}_{\mathbb{C}}\left(0,\left\lceil\boldsymbol{\sigma}^{2}\right\rfloor\right) .
$$

Notice that an heteroscedasticity of the noise is assumed, which means that the noise variance might vary over the microphones (i.e. the values in the vector $\sigma^{2}$ might be different).

Taking the expectation over the snapshots in Eq. (17) leads to the following decomposition of the theoretical CSM:

$$
\boldsymbol{S}_{p p}=\boldsymbol{L} \boldsymbol{L}^{H}+\left\lceil\boldsymbol{\sigma}^{2}\right\rfloor .
$$

There are several methods to solve this fitting problem. We propose in this paper two of them, based on a Bayesian approach. Two algorithms are used to find the maximum a posteriori estimates of the unknown parameters, namely each element of the matrix $\boldsymbol{L}$ and of the noise vector $\boldsymbol{\sigma}^{2}$ :

$$
\left(\hat{\boldsymbol{L}}, \hat{\boldsymbol{\sigma}}^{2}\right)=\operatorname{argmax}\left[\boldsymbol{L}, \boldsymbol{\sigma}^{2} \mid \hat{\boldsymbol{S}}_{p p}\right],
$$

where $[x \mid y]$ stands for the conditional PDF of $x$ given $y$.

\subsubsection{Expectation-Maximization algorithm}

The first procedure to solve (20) is an iterative algorithm that alternates between 2 steps at each iteration $i$ :

- performing the expectation of the complete-data log-posterior (i.e. including the missing values $\boldsymbol{S}_{c c}$ ) using the estimates of the parameters from the previous iteration $i-1$ (see p. 439-441 of Ref. 40):

$$
Q=\mathbb{E}\left\{\log \left[\boldsymbol{L}, \boldsymbol{S}_{c c}, \boldsymbol{\sigma}^{2} \mid \hat{\boldsymbol{S}}_{p p}, \boldsymbol{L}_{i-1}, \boldsymbol{S}_{c c_{i-1}}, \boldsymbol{\sigma}_{i-1}^{2}\right]\right\}
$$

- finding the parameters that maximize the expected value from the previous step:

$$
\left(\boldsymbol{L}_{i}, \boldsymbol{S}_{c c_{i}}, \boldsymbol{\sigma}_{i}^{2}\right)=\operatorname{argmax} Q
$$

These parameters are found making use of the old parameters to evaluate $\boldsymbol{S}_{c c_{i}}$ :

$$
\boldsymbol{S}_{c c_{i}}=\boldsymbol{I}_{\kappa}-\boldsymbol{B} \boldsymbol{L}_{i-1}+\boldsymbol{B} \hat{\boldsymbol{S}}_{p p} \boldsymbol{B}^{H}
$$

with

$$
\boldsymbol{B}=\boldsymbol{L}_{i-1}^{H}\left(\boldsymbol{L}_{i-1} \boldsymbol{L}_{i-1}^{H}+\left\lceil\boldsymbol{\sigma}^{2}\right\rfloor_{i-1}\right)^{-1} .
$$

Then, searching for the local maximum of $Q$ leads to the following updates of $\boldsymbol{L}$ and $\boldsymbol{\sigma}^{2}$ :

$$
\begin{aligned}
& \boldsymbol{L}_{i}= \hat{\boldsymbol{S}}_{p p} \boldsymbol{B}^{H} \boldsymbol{S}_{c c_{i}}^{-1} \\
& \boldsymbol{\sigma}_{i}^{2}=\operatorname{diag}\left(\left(\boldsymbol{I}_{M}-\boldsymbol{L}_{i} \boldsymbol{B}\right) \hat{\boldsymbol{S}}_{p p}\left(\boldsymbol{I}_{M}-\boldsymbol{L}_{i} \boldsymbol{B}\right)^{H}\right. \\
&\left.\quad+\boldsymbol{L}_{i}\left(\boldsymbol{I}_{\kappa}-\boldsymbol{B} \boldsymbol{L}_{i}\right) \boldsymbol{L}_{i}^{H}\right) .
\end{aligned}
$$

The reader can refer to the section 12.2.4 of Ref. 40 for detailed calculations and implementation of the Expectation-Maximization (EM) algorithm for PFA. The pseudo-code for this procedure is given in Algorithm 3. Note that, by construction, $\boldsymbol{S}_{c c}$ is positive semi-definite and therefore, so is the denoised CSM.

If the posterior is multimodal, the EM algorithm may converge toward a local maximum. Multiple strategies exist to avoid this situation, e.g. performing several EM iterations with different random initializations.

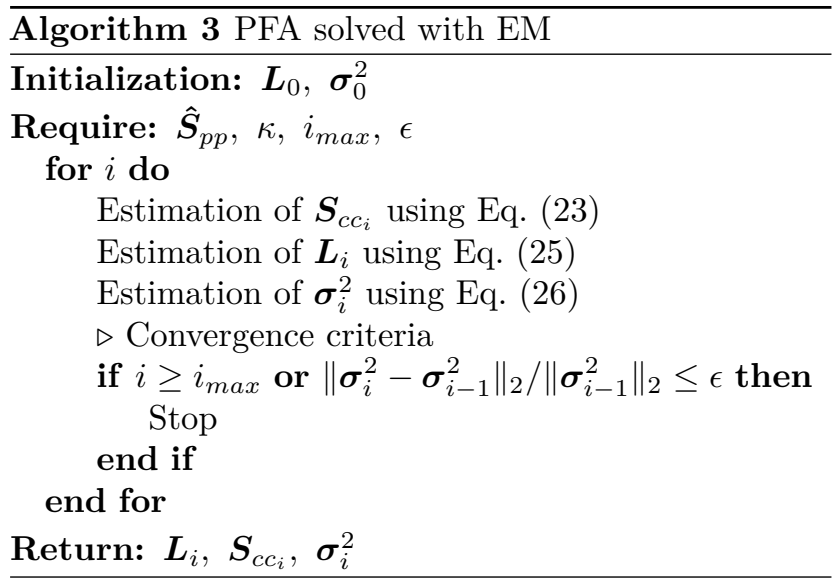

\subsubsection{Monte Carlo Markov Chain algorithm}

Another way to solve (20) is to use the Gibbs sampler [41], a Monte Carlo Markov Chain (MCMC) that consists in iterative draws in the marginal condition distributions of each parameters of the model until convergence. In this procedure, the sampling of the factors CSM is needed. Therefore, the unknown parameters are estimated by their maximum a posteriori:

$$
\left(\hat{\boldsymbol{L}}, \hat{\boldsymbol{\sigma}}^{2}, \hat{\boldsymbol{S}}_{c c}\right)=\operatorname{argmax}\left[\boldsymbol{L}, \boldsymbol{\sigma}^{2}, \boldsymbol{S}_{c c} \mid \hat{\boldsymbol{S}}_{p p}\right] .
$$

Unlike the EM algorithm, MCMC methods perform a global optimization and take advantages of the entire distribution available from the sampling. Therefore, MCMC can give a credible interval for each estimated parameter.

As a counterpart, MCMC are known to be far more computationally expensive than EM or other methods (see Table 4). 
In Table 1 can be found the prior PDFs assigned to each parameter of the model (17). First, the mixing matrix $\boldsymbol{L}$ is assumed to follow a centered Gaussian PDF, with a normalized variance, such that the energy of the signal part is only driven by the factors. The factors are supposed to be a priori independent and identically distributed, with the same energy $\gamma^{2}$ (also called homoscedasticity of the factors). The factor variance $\gamma^{2}$ is itself assigned a prior PDF in the form of an inverse Gamma law (written $\mathcal{I} \mathcal{G}$ ). This is a classical choice for variance parameters (see Ref. 42, p.42-43), since it has a positive support, it simplifies the maths thanks to its conjugacy with the Gaussian and finally, its shape and scale parameters can be tuned to specify different levels of prior information, from very precise to very vague.

The noise is supposed to be Gaussian, with a variance that may vary over the microphones (also called heteroscedasticity of the noise). This variance is distributed following an inverse Gamma law, for the same reason as for the factor variance. As MCMC enables more flexibility in the parametric model than EM, an extra unknown matrix $\lceil\boldsymbol{\alpha}\rfloor$ can be added, giving this model extension:

$$
\boldsymbol{p}_{j}=\boldsymbol{L}\lceil\boldsymbol{\alpha}\rfloor \boldsymbol{c}_{j}+\boldsymbol{n}_{j} \quad j=1, \ldots, N_{s},
$$

and taking the expectation over the snapshots:

$$
\boldsymbol{S}_{p p}=\boldsymbol{L}\lceil\boldsymbol{\alpha}\rfloor \boldsymbol{S}_{c c}\lceil\boldsymbol{\alpha}\rfloor \boldsymbol{L}^{H}+\left\lceil\boldsymbol{\sigma}^{2}\right\rfloor .
$$

This new diagonal matrix add weights to factor in a way that enforce sparsity on the latent factors. Indeed, these weights are assigned an exponential PDF (written $\mathcal{E}$ in Tab. 1) which is known to be a sparse prior. As this distribution has a great mass distributed around zero, it is prone to quickly shrink many values to zero. By doing so, even if the user overestimates the number of factors, unused factors will be set to zero and an optimal solution will be found with a better convergence rate.

All the posterior distribution that are required to implement the CSM-based Gibbs sampler are given in Appendix B. More details for the calculation of the posterior can be found in Ref. 43. The pseudo-code for the Gibbs sampler for PFA is shown in Algorithm 4, for which a thousand of iterations are performed and the returned estimate of denoised matrix results from the mean over the last 500 samples (in Algorithm 4, the number of iterations is denoted as $N_{\text {run }}$ ). Considering that these last samples tend to have a stationary, symmetric and unimodal distribution, the maximum a posteriori is assumed to be well estimated by the mean value.

Section 4.2 studies the influence of the chosen number of factors in the PFA models on the estimation error, through a numerical experiment. It is observed that

\begin{tabular}{l|ll}
\hline \hline Priors & \multicolumn{2}{|c}{ Hyper-priors } \\
\hline \hline$[\boldsymbol{c}]=\mathcal{N}_{\mathbb{C}}\left(0, \gamma^{2} \boldsymbol{I}_{\kappa}\right)$ & {$\left[\gamma^{2}\right]$} & $\mathcal{I}\left(a_{\gamma}, b_{\gamma}\right)$ \\
& {$\left[\boldsymbol{\sigma}^{2}\right]$} & $=$ \\
{$[\boldsymbol{n}]=\mathcal{N}_{\mathbb{C}}\left(0,\left\lceil\boldsymbol{\sigma}^{2}\right]\right)$} & $\mathcal{I} \mathcal{G}\left(\boldsymbol{a}_{\sigma}, \boldsymbol{b}_{\sigma}\right)$ & $=$ \\
{$[\boldsymbol{\alpha}]=\mathcal{E}\left(\boldsymbol{a}_{\alpha}\right)$} & \\
{$[\boldsymbol{L}]=\mathcal{N}_{\mathbb{C}}\left(0, \frac{\boldsymbol{I}_{M \kappa}}{\kappa}\right)$} & \\
\hline \hline
\end{tabular}

Table 1: Prior PDF assigned to each parameters of the PFA model solved with MCMC.

without weight (i.e. $\boldsymbol{\alpha}=1$ ), the reconstruction error given by the Gibbs sampler increases when the number of input factors is overestimated. On the contrary, the estimation error coming from the EM solver and the one from the MCMC solver fitting data on the sparse model (29) is minimal for any number of factors greater than the rank of the signal CSM (see Fig. 2).

Note that instead of adding some weights $\boldsymbol{\alpha}$, a strategy to promote sparsity of the model order would be to allow for heteroscedasticity of the factors in the model (17) as in Ref. 43. These two strategies are very similar: homoscedasticity is equivalent to a mixture of Gaussians with different variances following inverse Gamma laws, which is known to generate a Studentt marginal distribution for the factors $c$ (see Ref. 40, p.102-103), whereas the strategy of adding weights $\boldsymbol{\alpha}$ is equivalent to a mixture of Gaussians with different variances exponentially distributed, leading to a Laplace distribution for the product $\lceil\boldsymbol{\alpha}\rfloor \boldsymbol{c}[44]$. The Laplace and Student-t distributions are both heavy-tailed distributions, which are prompt to produce sparse solutions. In order to further enhance the sparsity of the solution, these two strategies may even be used simultaneously.

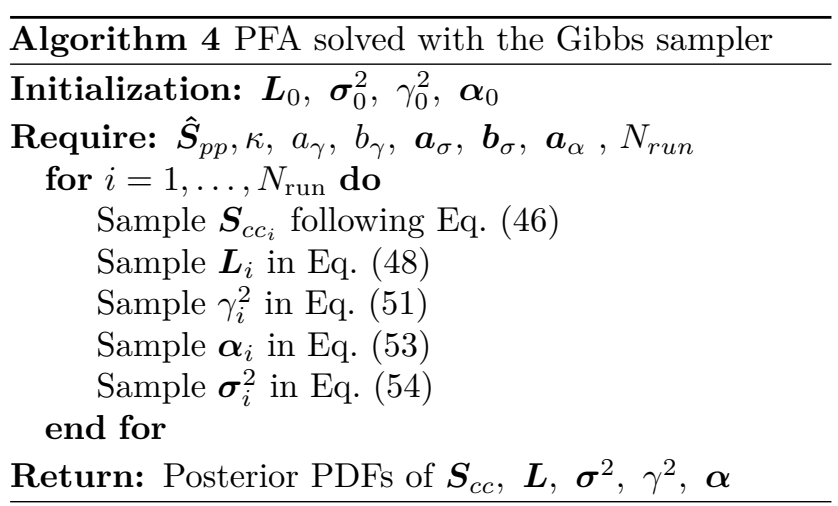

\section{Numerical experiments}

The denoising processes introduced above rely on different assumptions regarding the signal and noise properties. One can thus wonder what is the performance 
of each method depending on whether or not these assumptions hold true.

To answer this question, the denoising algorithms are tested on multiple CSMs, with varying properties, namely noise level, rank of the signal CSM and number of snapshots. The CSM are numerically simulated through the procedure described in the following section.

\subsection{Simulation of noisy CSM}

The way CSMs are simulated is inspired by a benchmark case from the aeroacoustic context, described in Ref. 45 and studied in Refs. 46 and 39. A line of free-field acoustic monopoles with spectra $\boldsymbol{q}$ radiates up to a circular array, which can be expressed as the linear system $\boldsymbol{a}=\boldsymbol{H} \boldsymbol{q}$, using the following Green's functions:

$$
\boldsymbol{H}_{m n}=\frac{e^{-\mathrm{j} k r_{m n}}}{4 \pi r_{m n}},
$$

with $k$ being the acoustic wavenumber $\frac{2 \pi f}{c_{0}}$ and $r_{m n}$ the distance between a source $n$ and a receiver $m$. The location of the sources and receivers is represented on Fig. 1.

Source spectra are independently drawn from a centered complex Gaussian distribution, with common variance $\sigma_{q}^{2}$ :

$$
\left[\boldsymbol{q}_{j}\right]=\mathcal{N}_{\mathbb{C}}\left(0, \sigma_{q}^{2} \boldsymbol{I}_{K}\right)
$$

where $j$ refers to the realization number and $K$ is the number of uncorrelated sources.

An independent Gaussian noise is then added to each signal $\boldsymbol{a}$ (see Eq. (2)):

$$
\begin{aligned}
& {\left[\boldsymbol{n}_{j}\right]=\mathcal{N}_{\mathbb{C}}\left(0,\left\lceil\boldsymbol{\sigma}^{2}\right\rfloor\right),} \\
& \text { with } \boldsymbol{\sigma}^{2}=\operatorname{diag}\left(\sigma_{q}^{2} \boldsymbol{H} \boldsymbol{H}^{H}\right) 10^{-\mathrm{SNR} / 10} .
\end{aligned}
$$

And finally, the CSM of measurements is estimated using Eq. (6) and the CSM without noise is also estimated in the same way: $\hat{\boldsymbol{S}}_{a a}=\frac{1}{N_{s}} \sum_{i=1}^{N_{s}} \boldsymbol{a}_{i} \boldsymbol{a}_{i}^{H}$. The objective of the denoising process is to recover this last quantity. The deviation of the denoised CSM (written $\tilde{\boldsymbol{S}}_{a a}$ ) from the noise-free simulation is evaluated looking at the reconstruction error of the diagonal elements, given by:

$$
\delta=\frac{\left\|\operatorname{diag}\left(\hat{\boldsymbol{S}}_{a a}\right)-\operatorname{diag}\left(\tilde{\boldsymbol{S}}_{a a}\right)\right\|_{2}}{\left\|\operatorname{diag}\left(\hat{\boldsymbol{S}}_{a a}\right)\right\|_{2}}
$$

where $\operatorname{diag}(\boldsymbol{A})$ is the vector containing the diagonal elements of $\boldsymbol{A}$ and $\|\cdot\|_{2}$ is the $\ell_{2}$ norm.

This reconstruction error is investigated for each denoising method and for varying parameters of the simulation:

- the rank of the signal matrix $\boldsymbol{S}_{a a}$, given by the number of uncorrelated monopoles,

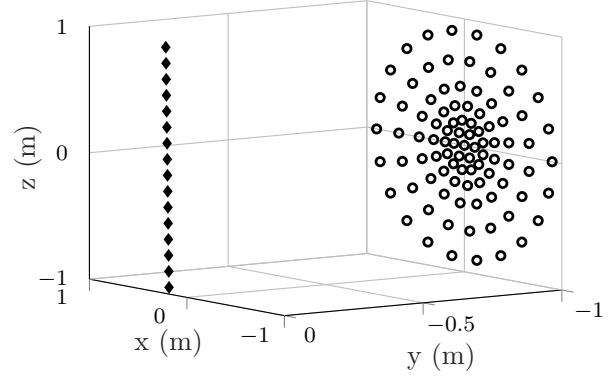

Figure 1: Receiver (o) and source $(\bullet)$ positions for acoustic field simulations, inspired by Ref. 45

- the noise level, given by a SNR varying from -10 to $10 \mathrm{~dB}$,

- the number of snapshots $N_{s}$ from 10 to $5.10^{4}$.

When a parameter is varied, the others remain constant, given by the default values from Tab. 2. Error curves are shown in Sec. 4.3.

\begin{tabular}{c|c}
\hline \hline Parameter & Default value \\
\hline \hline Frequency (invariant) & $f=15 \mathrm{kHz}$ \\
Sound velocity (inv.) & $340 \mathrm{~m} / \mathrm{s}$ \\
Number of receivers (inv.) & $M=93$ \\
Number of monopoles & $K=20$ \\
SNR & $\mathrm{SNR}=10 \mathrm{~dB}$ \\
Number of snapshots & $N_{s}=10^{4}$ \\
\hline \hline
\end{tabular}

Table 2: Default values for the numerical simulations.

The denoising performances of each method are mainly evaluated considering only the diagonal elements of the recovered matrix. The reason for this is that all the compared algorithms modify in a negligible way the off-diagonal terms. Illustrated explanations of this choice are given in section 4.4 .

\subsection{Initialization and input parameters for PFA}

In order to have a satisfying convergence rate, the EM and MCMC algorithms have to be properly initialized and the required priors have to be chosen appropriately.

\subsubsection{Initialization for PFA}

The initial values for PFA solved with EM and MCMC are given in Table 3. For the MCMC procedure, the noise is supposed to dominate the diagonal of the measured CSM $\boldsymbol{S}_{p p}$, so the noise variance is initialized with these diagonal elements. For the EM algorithm, the unknowns are empirically initialized close to zero. 


\begin{tabular}{l|l}
\hline \hline PFA-EM & PFA-MCMC \\
\hline $\boldsymbol{L}_{0}=10^{-16} \boldsymbol{J}_{M, \kappa}$ & $\boldsymbol{L}_{0}$ sampled in $\mathcal{N}_{\mathbb{C}}\left(0, \frac{\boldsymbol{I}_{M \kappa}}{\kappa}\right)$ \\
$\boldsymbol{\sigma}_{0}^{2}=10^{-16} \boldsymbol{I}_{M}$ & $\boldsymbol{\sigma}_{0}^{2}=\operatorname{diag}\left(\boldsymbol{S}_{p p}\right)$ \\
$\kappa=M-1$ & $\kappa=M-1$ \\
& $\boldsymbol{\alpha}_{0}=\operatorname{eig}_{\kappa}\left(\boldsymbol{S}_{p p}\right)$ \\
& $\gamma_{0}^{2}=\frac{1}{M} \operatorname{Trace}\left(\boldsymbol{S}_{p p}\right)$ \\
& $\boldsymbol{S}_{c c_{0}} \operatorname{sampled}$ in $\left[\boldsymbol{S}_{c c} \mid \boldsymbol{L}_{0}, \boldsymbol{\alpha}_{0}, \boldsymbol{\sigma}_{0}^{2}\right]$ \\
\hline \hline
\end{tabular}

Table 3: Initial values for PFA solved with EM and MCMC algorithms. $\boldsymbol{J}_{M, \kappa}$ is a $M \times \kappa$ matrix full of ones. The operator eig $_{\kappa}(\boldsymbol{A})$ refers to the $\kappa$ highest eigenvalues of $\boldsymbol{A}$, normalized in order to have $\sum \operatorname{eig}_{\kappa}(\boldsymbol{A})=1$.

\subsubsection{Initial choice for the number of factors $\kappa$}

In the PFA model, the maximum number of factors $\kappa$ that describes the acoustic field has to be set by the user. If this number is under-estimated (i.e. under the real number of independent source contributions), the acoustic field will not be fully described.

If the input number of factors is overestimated, the computational burden will be high and the convergence may be slow, until all the useless factors reach zero, depending on the initialization.

An illustration of this is given in Fig. 2. On this figure is plotted the reconstruction error of the diagonal element of the acoustical CSM, as a function of the input number of factors $\kappa$ for the EM and MCMC algorithms. For the two algorithms, the minimum error is obtained when the input $\kappa$ is equal to the real number of sources (20 in this example).

The EM algorithm is known to have a fast convergence if well initialized. That is why, even if $\kappa$ is overestimated, the reconstruction error remains low.

The solid gray line corresponds to the MCMC algorithm with the PFA model described by Eq. (29), without any update of the weights $\boldsymbol{\alpha}$, which means no sparsity enforced. In this case, if the initial number of factors is overestimated, the reconstruction error increases a bit because some useless factors are not totally switched off. On the contrary, when the sparsity in the PFA model is enforced with an update of the weights $\boldsymbol{\alpha}$, the effective number of sources is rapidly inferred from the data by weighting the useless factor, and the error remains low, even with a high $\kappa$.

\subsubsection{Prior parameters}

According to the Table 1 of the priors for PFA solved with MCMC, some constant parameters have to be set by the user. Here are the assigned values:

- $a_{\gamma}=b_{\gamma}=\boldsymbol{a}_{\sigma}=\boldsymbol{b}_{\sigma}=10^{-3}$ which corresponds to a very flat, and thus vague, prior on the source and noise variance.

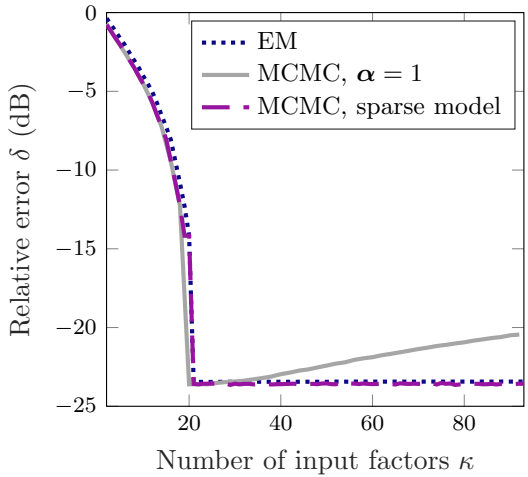

Figure 2: Relative reconstruction error of the signal CSM diagonal, as a function of the assumed number of factors in the PFA model. The minimum error is obtained when the input number of factor is equal to the number of sources used in the numerical experiment (default value is 20 ).

- $\boldsymbol{a}_{\alpha}=\frac{1}{\boldsymbol{\alpha}_{0}}$ so that $\boldsymbol{\alpha}_{0}$ is the probability mass center of the prior $[\boldsymbol{\alpha}]$.

\subsection{Results}

In this section, we compare the performance of each investigated approach to denoise the synthesized data, looking at the relative reconstruction error for different configurations of the numerical experiment described in Sec. 4. A list of the studied methods is given in Table 4 with a rough approximation of the computing time required by each method to denoise one $93 \times 93$ CSM, using non-optimized Matlab codes on a laptop.

\subsubsection{Comparison of the Diagonal Reconstruc- tion methods}

The three diagonal reconstruction methods described in Section 3.1 solve a very similar problem. We first compare them with one another. Error curves are given in Fig. 3, as a function of the rank of the signal CSM (a), the noise level (b) and the number of snapshots (c).

As expected, when no denoising is applied, the error is given by the opposite value of the SNR. The performance of each method mainly differs regarding the signal rank. As shown in Ref. 26, the error increases suddenly when the rank of the signal CSM is too high for the problem to remain identifiable. Convex optimization is less sensible to the increase of the number of sources, thanks to its faster convergence.

For 20 sources, sensitivity to noise level is the same for all the methods, and the error decreases linearly with an increasing SNR, and the same behavior is observed for a logarithmic increase of the number of snapshots.

As convex optimization runs faster and provides more 


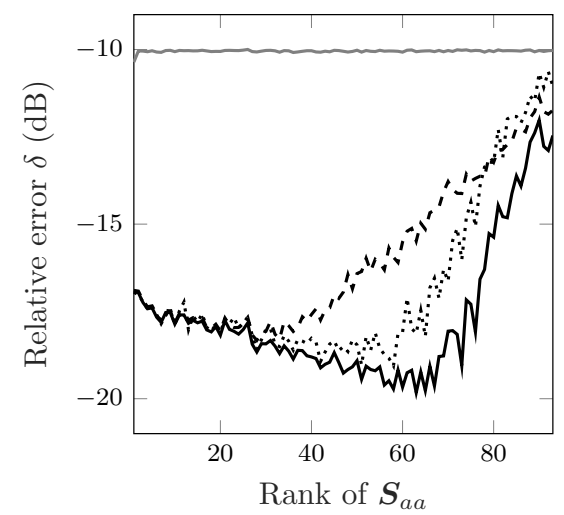

(a)

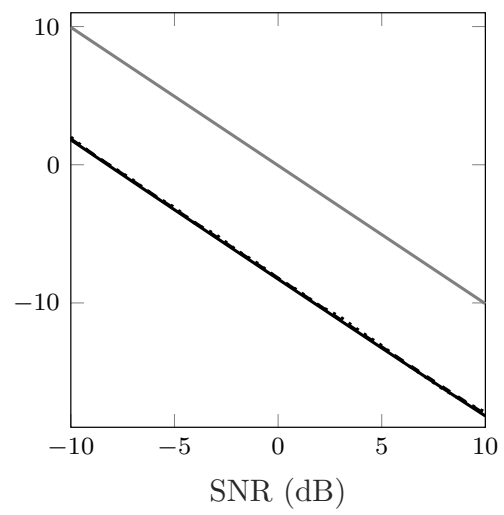

(b)

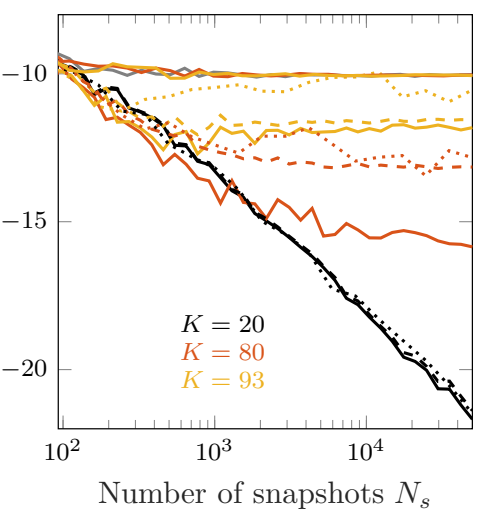

(c)

Figure 3: Relative reconstruction error of the diagonal terms of the signal CSM, as a function of the number of sources (a), SNR (b) and the number of snapshots (c). The diagonal reconstruction methods are: Alternating Projections (---), linear optimization $(\cdots \cdot$.$) , convex optimization (further referred to as DRec, -$ ), no denoising $(-)$.

\begin{tabular}{|c|c|c|}
\hline Denoising method & Acronym & $\begin{array}{l}\text { Computing } \\
\text { time }\end{array}$ \\
\hline Convex optimization & DRec & $1 \mathrm{sec}$ \\
\hline Linear optimization & & $60 \mathrm{sec}$ \\
\hline Alternating projections & & $3 \mathrm{sec}$ \\
\hline $\begin{array}{l}\text { Robust Principal Com- } \\
\text { ponent Analysis }\end{array}$ & RPCA & $0.5 \mathrm{sec}$ \\
\hline $\begin{array}{l}\text { Canonical Coherence } \\
\text { Analysis }\end{array}$ & $\mathrm{CCA}$ & $<0.1 \mathrm{sec}$ \\
\hline $\begin{array}{lr}\text { Probabilistic } & \text { Factor } \\
\text { analysis,solved } & \text { with } \\
\text { EM } & \end{array}$ & PFA-EM & $1 \mathrm{sec}$ \\
\hline $\begin{array}{l}\text { Probabilistic Factor } \\
\text { analysis, solved with } \\
\text { MCMC }\end{array}$ & PFA-MCMC & $\begin{array}{l}300 \text { sec for } \\
\kappa=92 \\
10 \text { sec for } \\
\kappa=10\end{array}$ \\
\hline
\end{tabular}

Table 4: List of the denoising methods and their approximate computing time to denoise one $93 \times 93 \mathrm{CSM}$, using non-optimized Matlab codes on a laptop.

accurate denoising, it is used for comparison with the other methods, referred to in the following as DRec (for Diagonal Reconstruction).

\subsubsection{Comparison of the other methods}

We now present the relative reconstruction error for all the other denoising methods, still considering only the diagonal elements of the acoustical CSM. In Fig. 4 is plotted the error as a function of the number of sources, SNR and number of averages, for the following methods:
- Convex optimization for diagonal reconstruction, referred to as DRec,

- RPCA with a constant regularization parameter close to $1 / \sqrt{M} \approx 0.1$, as suggested in Ref. 34,

- RPCA with the regularization parameter that gives the minimal error, written $\lambda_{\text {optimal }}$. Note that this optimal value will never be known in practice, since its calculation requires the knowledge of the groundtruth CSM $\boldsymbol{S}_{a a}$,

- CCA with both thresholding of the canonical coherences and adaptive iteration count,

- PFA solved with the EM algorithm, referred to as PFA-EM,

- PFA solved with the MCMC algorithm, referred to as PFA-MCMC,

- no denoising applied.

The results from the PFA denoising achieved with EM and MCMC are very similar because both methods rely on the formulation of a very similar inverse problem. However, when the number of sources is high, EM performs better than MCMC, because the latter makes a stronger assumption on low-rankness of the signal CSM. Moreover, for very low number of sources, or low number of snapshots, EM does not converge toward an optimal solution, whereas MCMC, being a global optimization algorithm, converges better.

Concerning RPCA, one can see that the selection of a regularization parameter can have a strong impact on the denoising performance, especially when the number of sources increases. The parameter $\lambda=0.1$ proposed by Wright is tuned for low rank signal matrix. When the number of sources increases, the regularization parameter must also increase to keep the balance between 


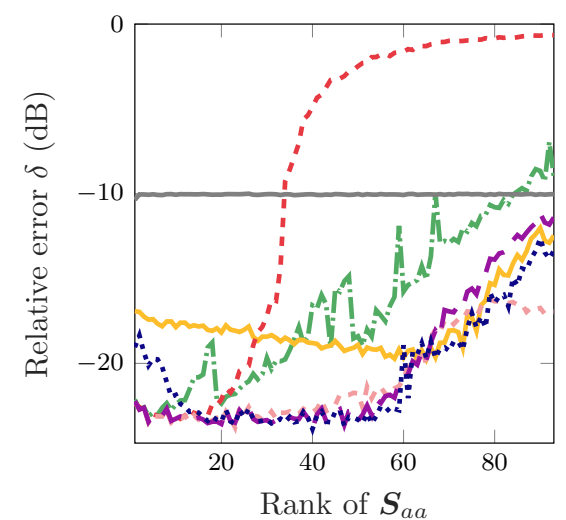

(a)

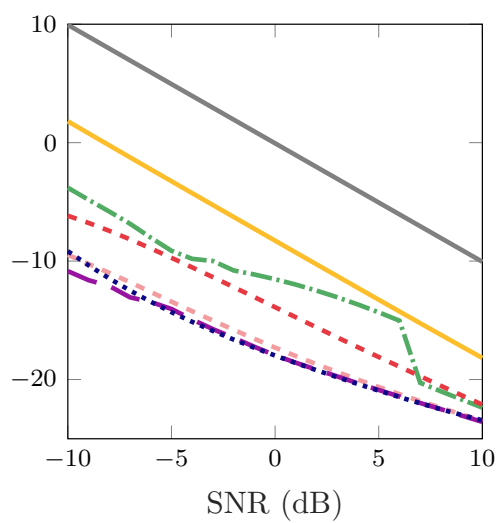

(b)

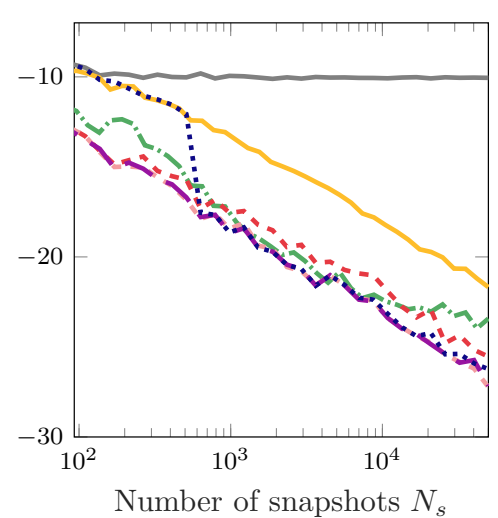

(c)

Figure 4: Relative reconstruction error of the diagonal terms of the signal CSM, as a function of the number of sources (a), SNR (b) and the number of snapshots (c). The denoising methods are: DRec (-), RPCA with

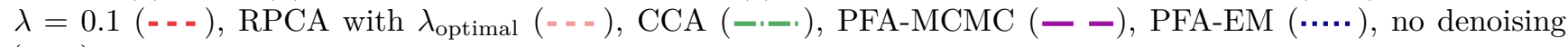
$(-)$.

the low-rankness of the signal CSM and sparsity of the noise CSM.

When proper assumptions are fulfilled (low rank signal CSM and high number of snapshots), the PFA solution is similar to one given by RPCA when using the optimal regularization parameter, whereas the DRec error is most of the time $5 \mathrm{~dB}$ higher.

The reconstruction error given by CCA is very similar to those presented in Ref. 39 since the simulated case is nearly the same. The number of iterations automatically selected from the number of significant canonical coherences is also comparable to Ref. 39. This varying number of iterations induces significant discontinuities on the CCA error plotted versus the number of sources, which can be corrected by an appropriate tuning of the empirical thresholding values and iteration count criterion. Note that the CCA method generally suffers from a bias error - due to the fact that the square root matrices $\boldsymbol{S}_{x x}^{-1 / 2}$ and $\boldsymbol{S}_{y y}^{-1 / 2}$ used in Algorithm 2 carry over the presence of noise - which probably explains why it has an error a few $\mathrm{dB}$ higher than PFA and RPCA.

\subsection{About the denoising of the off- diagonal terms}

All the studied methods make the assumption that the noise CSM is diagonal or nearly diagonal. Consequently, the extra-diagonal elements are expected to be almost unchanged by the denoising process.

To verify this fact, we can compare the relative error of the off-diagonal elements, defined as follows:

$$
\delta_{\text {off }}=\frac{\left\|\hat{\boldsymbol{S}}_{a a}-\tilde{\boldsymbol{S}}_{a a}-\left[\operatorname{diag}\left(\hat{\boldsymbol{S}}_{a a}-\tilde{\boldsymbol{S}}_{a a}\right)\right]\right\|_{F}}{\left\|\hat{\boldsymbol{S}}_{a a}-\left[\operatorname{diag}\left(\hat{\boldsymbol{S}}_{a a}\right)\right]\right\|_{F}},
$$

where $\|\cdot\|_{F}$ is the Frobenius norm.

The relative error curves of the cross-spectra are plotted in Fig. 5, for the denoising performed with PFA, RPCA and CCA, always from the same numerical experiments. On this figure, one can see that PFA and RPCA denoising do not significantly change the off-diagonal terms, except that PFA provides a slight denoising for very low SNR. Neither RPCA nor PFA gives a worse error than without any denoising. This is not the case for CCA which modifies the cross-spectra, except when the number of sources is lower than 10 and when the number of snapshots is lower than 6000 .

\section{Experimental application}

The denoising methods are now compared on data acquired in a wind-tunnel, so as to see how they behave with a real turbulent boundary layer noise (TBL).

\subsection{Experimental setup}

The experiment is conducted in a closed-section wind tunnel at École Centrale de Lyon (LMFA laboratory), shown in Fig. 6(a). Three acoustic sources are mounted in the ceiling of the test-section, arranged in a dipole and a monopole, spaced by $20 \mathrm{~cm}$, excited by two uncorrelated white noises of comparable amplitude. Wallpressure fluctuations are measured using an array of 73 


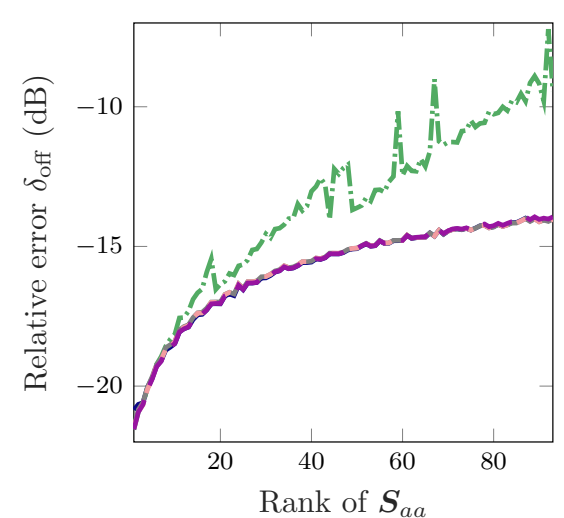

(a)

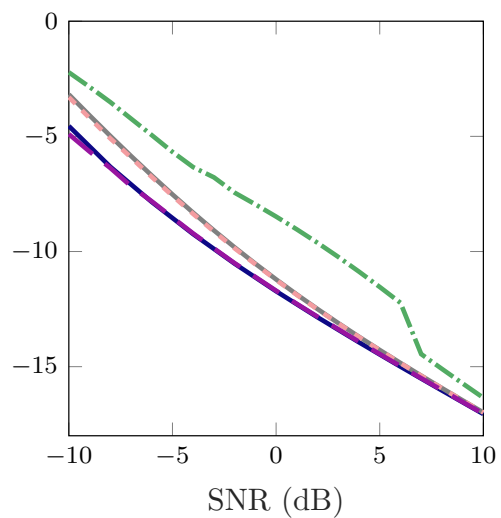

(b)

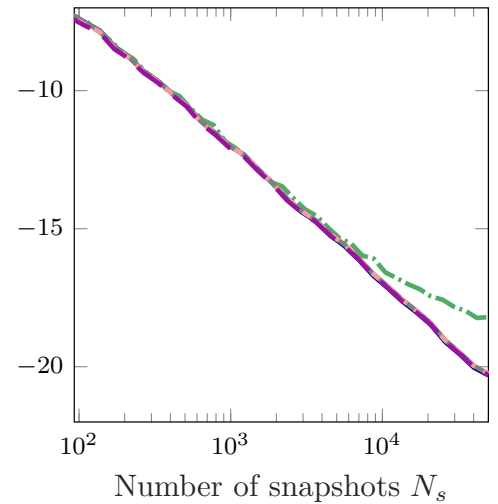

(c)

Figure 5: Relative reconstruction error of the signal CSM cross-spectra obtained from 3 denoising methods: RPCA with $\lambda_{\text {optimal }}(---)$, PFA-MCMC $(--)$, PFA-EM (.....), CCA (--_.) and without denoising $(-)$, as a function of the number of sources (a), SNR (b) and number of snapshots (c).

MEMS microphones in a spiral arrangement, located in the floor of the tunnel (see Fig. 6(b) and Fig. 6(c)). The microphone inter-spacing varies from $0.2 \mathrm{~cm}$ to $27.4 \mathrm{~cm}$.

A first measurement without flow is performed to be used as a baseline for the acoustical field CSM. The measurements to be denoised are conducted with a flow speed of $20 \mathrm{~m} / \mathrm{s}$ and with the acoustic sources switched on. The duration of the acquired signal is $30 \mathrm{~s}$ and the CSM are computed with a $16 \mathrm{~Hz}$ resolution, and a Hanning windows with $66 \%$ of overlapping, which is equivalent to about 994 effective snapshots.

\section{$5.2 \quad$ Results}

\subsubsection{Denoised autospectra}

Figure 7(a) shows the autospectra, averaged over the microphones, of all the denoised CSMs, along with the baseline source autospectrum and the $95 \%$ credible interval provided by the PFA-MCMC denoising. As the noise may vary over the microphones, the average autospectra may not be fully representative of the denoising level. Therefore, a denoising error for each method is also given in Fig. 7(b), which illustrates the distance from each microphone autospectrum to the corresponding baseline autospectrum as follows:

$$
\delta=\frac{\left\|\operatorname{diag}\left(\boldsymbol{S}_{a a}^{\star}\right)-\operatorname{diag}\left(\tilde{\boldsymbol{S}}_{a a}\right)\right\|_{2}}{\left\|\operatorname{diag}\left(\boldsymbol{S}_{a a}^{\star}\right)\right\|_{2}},
$$

with $\tilde{\boldsymbol{S}}_{a a}$ is the denoised CSM and $\boldsymbol{S}_{a a}^{\star}$ is the baseline source CSM.

It not possible to have $\tilde{\boldsymbol{S}}_{a a}=\boldsymbol{S}_{a a}^{\star}$ in general, first because of the estimation error due to the limited number of snapshots and second, because of the convection effect. Indeed, the denoised CSM contains the acoustical part subjected to a convection effect, which is not compensated by the denoising process, whereas the baseline source CSM comes from measurements without any convection effect. Therefore, even after an optimal denoising, the denoising error should be limited by these two thresholds, which are numerically evaluated further below.

The major differences between the experimental data and the previous numerical simulations concern the noise properties. In the real measurements, the noise is generated by the pressure fluctuations caused by the turbulent boundary layer and dominates the data, leading to a very poor signal-to-noise ratio (from more than $-20 \mathrm{~dB}$ at low frequencies to $-5 \mathrm{~dB}$ at high frequencies). Moreover, the TBL noise is highly correlated over the microphones in the low frequency range, which does not fulfill the requirements of the denoising methods investigated in the present work. Therefore, low performance is expected from every methods at low frequencies.

CCA and PFA associate all the coherent field to the signal CSM, possibly including the one from the TBL noise. Therefore, the signal CSM is overestimated in the frequency range where the TBL noise is highly correlated over the microphones. RPCA provides a more efficient denoising below $1.2 \mathrm{kHz}$, thanks to the value of the regularization parameter $(\lambda=0.1)$, which drives the solution to a favorable low-rankness of the signal CSM, and slightly prevent the autospectra from being overestimated.

The denoising performance provided by PFA-EM is very variable over frequencies, because the algorithm converges to local maximima that depend on the initial- 


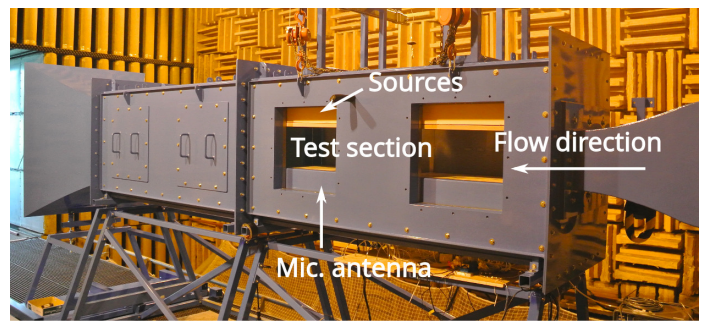

(a) Picture of the facility.

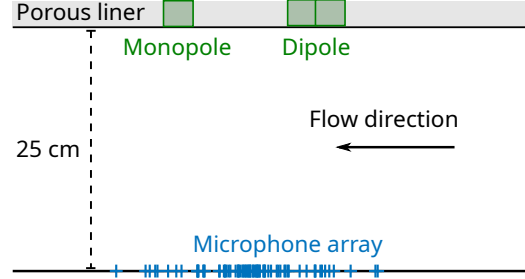

(b) Sketch of the test section (side view).

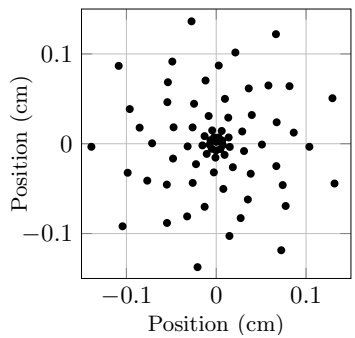

(c) Design of the MEMS microphone array.

Figure 6: Description of the facility for the wind-tunnel tests.

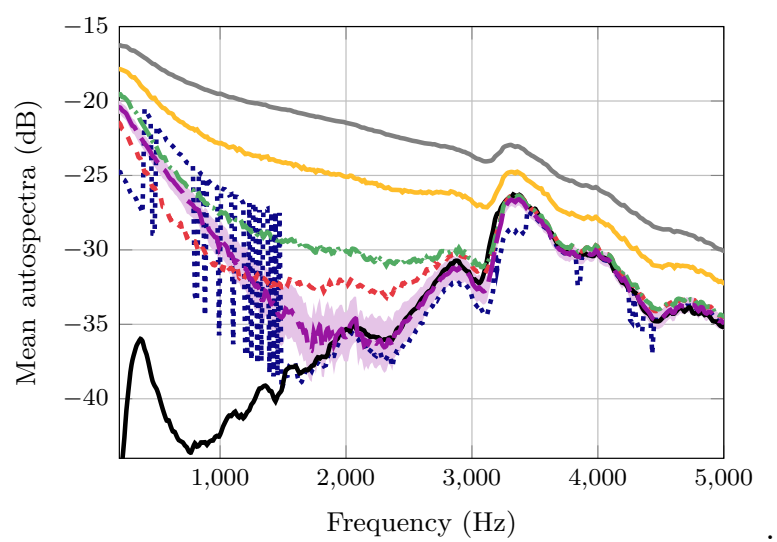

(a) Autospectra from the denoised CSMs, averaged over the microphones

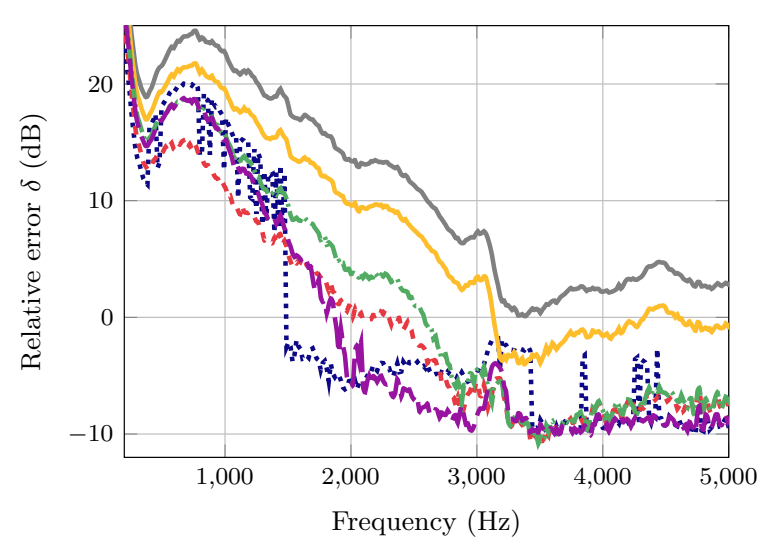

(b) Denoising error based on the measurement of the sources without flow (from Eq. (35)).

Figure 7: CSM denoised with DRec ( - $)$, RPCA with $\lambda=0.1$ (- - - ), CCA (-.-.), PFA-MCMC (- - ), PFA-EM $(\cdots .$.$) , and the CSM not denoised (-). On (a) is also plotted the source mean autospectrum (-)$ and the $95 \%$ credible interval for PFA-MCMC ( $)$.

ization and the choice of the stopping criteria. Below $1.5 \mathrm{kHz}$, PFA-EM provides a very unstable solution, with a rather high denoising error compared to those from the other methods.

In the low frequency range, the efficiency of CCA could be improved by a better thresholding of the canonical coherences, but the appropriate thresholding is hard to set in practice when no information about the real source CSM is available. The performance of PFAMCMC could also be improved by taking into account a CSM fitting a TBL model such as Corcos [47] in Eq. (29), with known or inferred TBL parameters. This can be done by simply adding one or several sampling steps in the Gibbs sampler.

Between 2 and $3 \mathrm{kHz}$, PFA-MCMC provides an estimate of the mean source autospectra very close to the measurement without noise, thanks to a sparse model that leads to an exact estimation of 2 uncorrelated components in the acoustic field.

Up to $5 \mathrm{kHz}$, the autospectra denoised with the diagonal reconstruction method remain overestimated of at least $3 \mathrm{~dB}$. Above $3.5 \mathrm{kHz}, \mathrm{PFA}, \mathrm{CCA}$ and RPCA provide a similar amount of denoising, but the denoising error is limited by the convection effect on the acoustic propagation. Indeed, the effect of the flow on the acoustic propagation imposes a lower bound for the denoising error of Eq. (35). This bound can be evaluated numerically, by simulating the source propagation with and without convection.

Knowing the positions for the sources and microphones from the experimental setup, two measured CSM are simulated. First, the baseline source CSM is simulated using free-field Green functions (see Eq. (30)). Then, another acoustic CSM is simulated using a convected propagation:

$$
\boldsymbol{H}_{m n}^{\text {conv }}=\frac{e^{j k \Delta r_{m n}}}{4 \pi \sqrt{\left(\boldsymbol{M} \cdot \boldsymbol{r}_{m n}\right)^{2}+\beta^{2}\left|\boldsymbol{r}_{m n}\right|^{2}}}
$$

with $\Delta r_{m n}=\frac{1}{\beta^{2}}\left(-\boldsymbol{M} \cdot \boldsymbol{r}_{m n}+\sqrt{\left(\boldsymbol{M} \cdot \boldsymbol{r}_{m n}\right)^{2}+\beta^{2}\left|\boldsymbol{r}_{m n}\right|^{2}}\right)$ and where $\boldsymbol{M}$ is the Mach number vector, $\beta^{2}=1-|\boldsymbol{M}|^{2}$, 


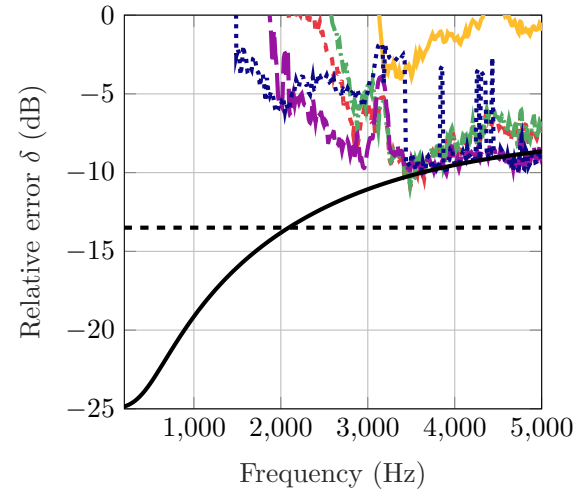

Figure 8: Same error curve as on Fig. 7(b), along with the error due to the convection effect on the acoustic field (black solid line) and the estimation error of the CSM (black dashed line).

$\boldsymbol{r}_{m n}$ is the difference between the microphone $m$ and the source $n$ position vectors and - indicates a dot product. The two simulated CSMs are then injected in the Eq. (35) ( $\tilde{\boldsymbol{S}}_{a a}$ being the convected acoustic CSM and $\boldsymbol{S}_{a a}^{\star}$ the free-field one) and the error is plotted in Fig. 8. This error depends on the frequency because of the directivity of the dipole. On this figure is also plotted the estimation error of the CSM due to the finite number of snapshots (994 for the experimental test cases). One can see that the denoising error from $3.5 \mathrm{kHz}$ is clearly limited by the convection effect on the denoised spectra, but not by the estimation error.

\subsubsection{Rank of the denoised CSMs}

As several denoising methods rely on a low-rankness assumption for the acoustic CSM, Fig 9 shows the eigenvalues of the denoised CSM at 3 different frequencies. On this Figure, one can see that RPCA does not preserve the positive-semidefiniteness of the denoised CSM. A positivity constrain could be added to the RPCA problem, as proposed in Ref. 36. It is also visible that DRec performs a reduction of the eigenvalues, until the smallest one reaches zero.

An overview of the eigenvalues of the denoised CSM against frequency is also given in Fig. 10. The number of significant eigenvalues are plotted on this figure an eigenvalue is arbitrary considered significant if it is greater than $1 \%$ of the highest one. At low frequencies, all the methods overestimate the rank of the denoised CSM, because of the correlation of the TBL noise over the microphones. Above $2200 \mathrm{~Hz}$, PFA-MCMC provides a CSM with exactly 2 significant eigenvalues, whereas PFA-EM often provides only one significant eigenvalue (which is related to the underestimation of the mean autospectra on Figure 7(a)). The CSMs denoised by CCA all shows a jump in their eigenvalues, due to the thresholding step during the denoising process. In general, the experimental setup with 2 uncorrelated sources is favorable to the PFA-MCMC approach which is able, thanks to its strong sparsity contraint, to provide a very low-rank CSM.

\section{Conclusion}

This work offers an overview of some methods for the denoising of the CSM in the framework of multi-sensor acoustic measurements.

The three diagonal reconstruction methods give very comparable results with varying computational costs. The diagonal reconstruction method with the lowest computational cost is the one based on the convex optimization, but its performance is limited when the number of sources is very low, as shown by the numerical and wind-tunnel experiments.

RPCA and CCA provide a higher noise reduction, but their performance relies on the setting of empirical parameters, respectively a regularization parameter and a thresholding of the canonical coherences.

Finally, the Bayesian-based method PFA allows for a high level of denoising, assessed on numerical simulations and on measurements in presence of a strong TBL noise, as shown in the present study and in Ref. 48. The main drawback of the PFA method is its high computational cost, yet this is balanced by some benefits, among which:

- the denoised CSM is written as a linear combination of a very limited number of components, which might result in a high data-compression,

- the Gibbs sampler returns a credible interval for each inferred parameter,

- the Bayesian framework is very flexible and any prior information about the acoustic or the noise CSM can be considered. For example, an extension of the model could take into account a correlation structure in the noise CSM.

\section{Acknowledgements}

This work was performed within the framework of the Labex CeLyA of the Université de Lyon, within the program 'Investissements d'Avenir' (ANR-10-LABX0060/ANR-16-IDEX-0005) operated by the French National Research Agency (ANR). This work was also performed in the framework of Clean Sky 2 Joint Undertaking, European Union (EU), Horizon 2020, CS2-RIA, ADAPT project, Grant agreement no 754881. 


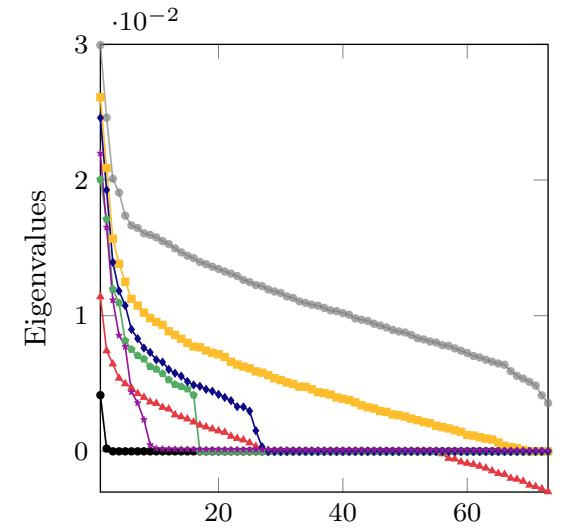

Eigenvalue number

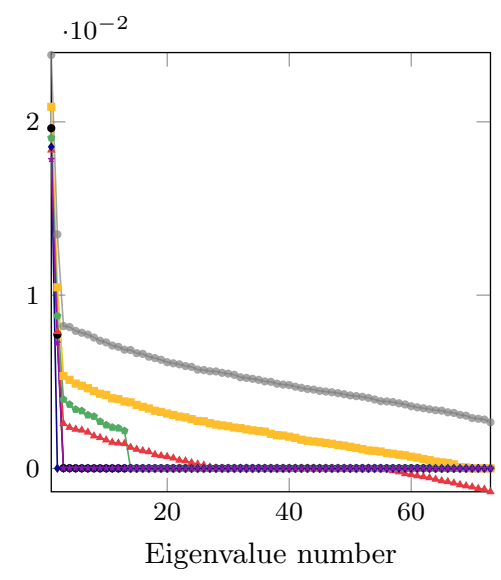

(b) At $2512 \mathrm{~Hz}$

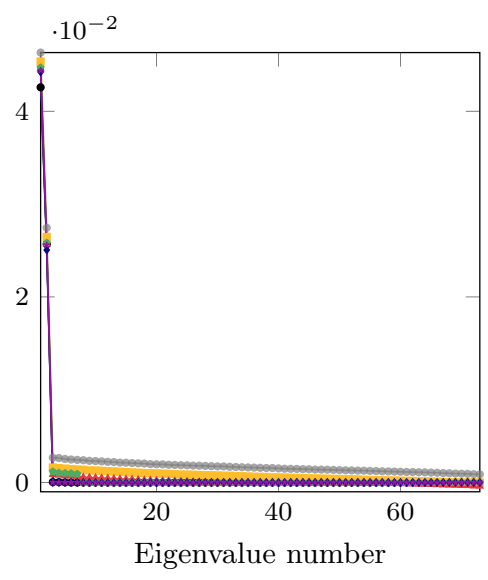

(c) At $4000 \mathrm{~Hz}$

Figure 9: Eigenvalues of the CSM denoised with: DRec $(\backsim-n)$, RPCA with $\lambda=0.1(\Delta \Delta)$, CCA ( $\cdots)$, PFA-MCMC

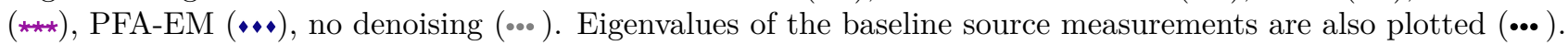

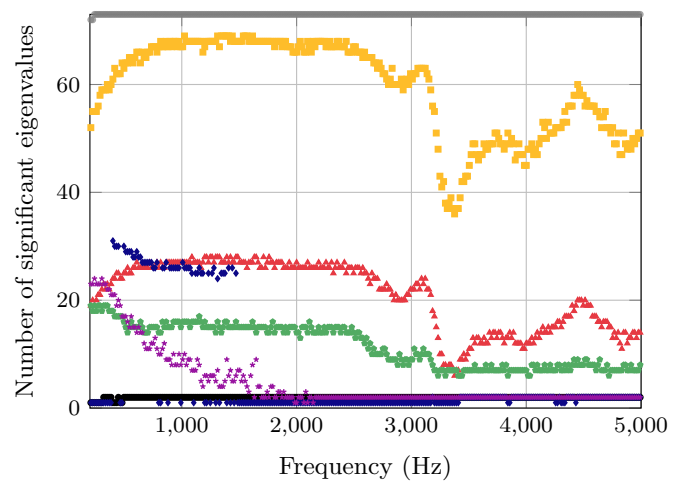

Figure 10: Number of significant eigenvalues of the CSM denoised with DRec $(\backsim \pi)$, RPCA with $\lambda=0.1(\Delta \Delta)$, CCA $(\cdots)$, PFA-MCMC (**), PFA-EM (**), also of the CSM not denoised (...) and of the baseline source CSM (...). The significant eigenvalues are those greater than $1 \%$ of the highest eigenvalue.

\section{A Relation to Chung's method}

In an early paper, Chung[2] introduced a method for rejecting flow-noise in measured auto-spectra, in the special scenario where three microphones measure a group of completely coherent sources - treated as an equivalent single source. In lines of the present paper, the method assumes flow-noises to be mutually uncorrelated at the microphones. By making use of the coherence functions between microphones, it returns denoised auto-spectra at each of the three microphones. As far as the authors know, this is probably one of the first attempts to denoise the CSM. The principle of the method is resumed using the notation of the present paper. Let $\hat{S}_{p_{i} p_{j}}$ denote the measured cross-spectrum between the pressure signals $p_{i}$ and $p_{j}$ measured by a pair of microphones and $S_{a_{i} a_{j}}$ the corresponding theoretical signal cross-spectrum $\left(\hat{S}_{p_{i} p_{j}}\right.$ and $S_{a_{i} a_{j}}$ are the elements in cell $(i, j)$ of the CSMs $\hat{\boldsymbol{S}}_{p p}$ and $\boldsymbol{S}_{a a}$, respectively). After some lengthy calculations, Chung arrived at the following estimator of the signal cross-spectrum,

$$
\hat{S}_{a_{i} a_{i}}=\hat{S}_{p_{i} p_{i}} \frac{\gamma_{i k} \gamma_{l i}}{\gamma_{l k}}, \quad k \neq l \neq i, \quad i=1,2,3
$$

with $\gamma_{i j} \triangleq \hat{S}_{p_{i} p_{j}} / \sqrt{\hat{S}_{p_{i} p_{i}} \hat{S}_{p_{j} p_{j}}}$ the coherence function between $p_{i}$ and $p_{j}$. This results arrives readily when reformulating Chung's problem by means of Eq. (19),

$$
\boldsymbol{S}_{p p}=\boldsymbol{L} \boldsymbol{L}^{H}+\left\lceil\boldsymbol{\sigma}^{2}\right\rfloor
$$

where $\boldsymbol{L}$ is now a column vector of an arbitrary dimension $M \geqslant 3$. It then comes that $\hat{S}_{p_{i} p_{j}} \approx L_{i} L_{j}^{*}$ and, therefore,

$$
\begin{array}{r}
\hat{S}_{a_{i} a_{i}}=\hat{S}_{p_{i} p_{i}} \frac{\gamma_{i k} \gamma_{l i}}{\gamma_{l k}}=\frac{\hat{S}_{p_{i} p_{k}} \hat{S}_{p_{l} p_{i}}}{\hat{S}_{p_{l} p_{k}}} \approx\left|L_{i}\right|^{2} \\
i=1, \ldots, M .
\end{array}
$$

This proves in one line that $\hat{S}_{a_{i} a_{i}}$, as given in Eq. (37), is a valid estimator of the signal cross-spectrum. One drawback of Chung's method is to be limited to the use of only three microphones out of many more possibly available. When more than three transducers are available, it requires the selection of an arbitrary subset of three microphones in order to apply Eq. (37). Because of its lack of generality, it will not be compared with the other methods in the experimental sections. 


\section{B Posterior distributions for the Gibbs sampler}

Using the Bayes rule, the posterior distribution of each parameter is given by:

$$
\begin{aligned}
{[\theta \mid \infty] \propto } & \underbrace{\prod_{i}\left[\mathrm{i}^{\text {th }} \text { child of } \theta \mid \text { Parents of } \mathrm{i}^{\text {th }} \text { child }\right]}_{\text {Likelihood }} \\
& \times \underbrace{[\theta \mid \text { Parents of } \theta]}_{\text {Prior }}
\end{aligned}
$$

where " $\infty$ " is to be understood as "conditioned to all the other variables of the model". In this expression, the children of $\theta$ are all the parameters of the model that directly depend on $\theta$, and the parents of $\theta$ are all the parameters on which $\theta$ depends.

\section{B.1 Expression of the likelihood of the data}

According to the Central Limit theorem applied to Fourier coefficients, the likelihood function of the measured data tends to be Gaussian:

$$
\left[\boldsymbol{p}_{j} \mid \infty\right]=\mathcal{N}_{\mathbb{C}}\left(\boldsymbol{L}\lceil\boldsymbol{\alpha}\rfloor \boldsymbol{c}_{j},\left\lceil\boldsymbol{\sigma}^{2}\right\rfloor\right)
$$

for the $j^{\text {th }}$ measurement.

\section{B.2 Sampling of $\boldsymbol{S}_{c c}$}

From the expressions of the likelihood (41) and the prior in Table 1, the posterior of the factors $\boldsymbol{c}$ is:

$$
\begin{aligned}
{\left[\boldsymbol{c}_{j} \mid \infty\right] } & \propto\left[\boldsymbol{p}_{j} \mid \infty\right]\left[\boldsymbol{c}_{j}\right] \\
& \propto \mathcal{N}_{\mathbb{C}}\left(\boldsymbol{L}\lceil\boldsymbol{\alpha}\rfloor \boldsymbol{c}_{j},\left\lceil\boldsymbol{\sigma}^{2}\right\rfloor\right) \mathcal{N}_{\mathbb{C}}\left(\mathbf{0}, \gamma^{2} \boldsymbol{I}_{\kappa}\right)
\end{aligned}
$$

Using the multiplication rule of Gaussians (see for example Ref. 49) directly gives

$$
\left[\boldsymbol{c}_{j} \mid \infty\right] \propto \mathcal{N}_{\mathbb{C}}\left(\boldsymbol{\mu}_{c_{j}}, \boldsymbol{\Omega}_{c}\right)
$$

$$
\text { with } \quad \boldsymbol{\Omega}_{c}=\left(\lceil\boldsymbol{\alpha}\rfloor \boldsymbol{L}^{H}\left\lceil\boldsymbol{\sigma}^{-2}\right\rfloor \boldsymbol{L}\lceil\boldsymbol{\alpha}\rfloor+\gamma^{-2} \boldsymbol{I}_{\kappa}\right)^{-1}
$$$$
\text { and } \boldsymbol{\mu}_{c_{j}}=\boldsymbol{\Omega}_{c}\lceil\boldsymbol{\alpha}\rfloor \boldsymbol{L}^{H}\left\lceil\boldsymbol{\sigma}^{-2}\right\rfloor \boldsymbol{p}_{j}
$$

In order to built a CSM-based Gibbs sampler, the same approach as in Ref. 43 is followed. As $\boldsymbol{c}_{j} \mid \infty$ is Gaussian, it can be written

$$
\boldsymbol{c}_{j} \mid \infty=\boldsymbol{\mu}_{c_{j}}+\boldsymbol{x}_{j} \quad \text { with } \quad\left[\boldsymbol{x}_{j}\right]=\mathcal{N}_{\mathbb{C}}\left(\mathbf{0}, \boldsymbol{\Omega}_{c}\right) .
$$

Then,

$$
\boldsymbol{S}_{c c}=\frac{1}{N_{s}} \sum_{j=1}^{N_{s}} \boldsymbol{\mu}_{c_{j}} \boldsymbol{\mu}_{c_{j}}^{H}+\frac{1}{N_{s}} \sum_{j=1}^{N_{s}} \boldsymbol{x}_{j} \boldsymbol{x}_{j}^{H}+\frac{2}{N_{s}} \sum_{j=1}^{N_{s}} \boldsymbol{x}_{j} \boldsymbol{\mu}_{c_{j}}^{H} .
$$

Since $\boldsymbol{x}_{j}$ and $\boldsymbol{\mu}_{c_{j}}$ are independent random variables, the last terms tends to zero, and then

$$
\begin{aligned}
\boldsymbol{S}_{c c} \mid \infty \approx & \boldsymbol{\Omega}_{c}\lceil\boldsymbol{\alpha}\rfloor \boldsymbol{L}^{H}\left\lceil\boldsymbol{\sigma}^{-2}\right\rfloor \boldsymbol{S}_{p p}\left\lceil\boldsymbol{\sigma}^{-2}\right\rfloor^{H} \boldsymbol{L}\lceil\boldsymbol{\alpha}\rfloor \boldsymbol{\Omega}_{c}^{H} \\
& +\frac{1}{N_{s}} \boldsymbol{W}_{c}
\end{aligned}
$$

where $\boldsymbol{W}_{c}$ is a random matrix that follows a complex Wishart distribution, with $N_{s}$ degrees of freedom and variance matrix $\boldsymbol{\Omega}_{c}$.

\section{B.3 Sampling of $L$}

The sampling of $\boldsymbol{L}$ is made using a vectorized form of $\boldsymbol{L}$, written $\boldsymbol{\lambda}=\operatorname{vec}(\boldsymbol{L})$. The posterior of $\boldsymbol{\lambda}$ is given by

$$
\begin{aligned}
{[\boldsymbol{\lambda} \mid \infty] } & \propto \prod_{j=1}^{N_{s}}\left[\boldsymbol{p}_{j} \mid \infty\right][\boldsymbol{\lambda}] \\
& \propto \mathcal{N}_{\mathbb{C}}\left(\boldsymbol{L}\lceil\boldsymbol{\alpha}\rfloor \boldsymbol{c}_{j},\left\lceil\boldsymbol{\sigma}^{2}\right\rfloor\right) \mathcal{N}_{\mathbb{C}}\left(0, \frac{\boldsymbol{I}_{M \kappa}}{\kappa}\right) .
\end{aligned}
$$

Using the fact that

$$
\boldsymbol{p}_{j}=\operatorname{vec}\left(\boldsymbol{L}\lceil\boldsymbol{\alpha}\rfloor \boldsymbol{c}_{j}\right)+\boldsymbol{n}_{j}=\left(\lceil\boldsymbol{\alpha}\rfloor \boldsymbol{c}_{j}^{T} \otimes \boldsymbol{I}_{M}\right) \boldsymbol{\lambda}+\boldsymbol{n}_{j},
$$

and some properties of the Kronecker product $\otimes$ (see for example Ref. 50, p. 59-60) leads to:

$$
[\boldsymbol{\lambda} \mid \infty] \propto \mathcal{N}_{c}\left(\boldsymbol{\mu}_{\lambda}, \boldsymbol{\Omega}_{\lambda}\right),
$$

$$
\text { with } \boldsymbol{\Omega}_{\lambda}^{-1}=\lceil\boldsymbol{\alpha}\rfloor \boldsymbol{S}_{c c}^{*}\lceil\boldsymbol{\alpha}\rfloor \otimes\left\lceil\boldsymbol{\sigma}^{-2}\right\rfloor+\kappa \boldsymbol{I}_{M \kappa}
$$$$
\text { and } \boldsymbol{\mu}_{\lambda}=\boldsymbol{\Omega}_{\lambda} \operatorname{vec}\left(\left\lceil\boldsymbol{\sigma}^{-2}\right\rfloor \boldsymbol{S}_{p c}\lceil\boldsymbol{\alpha}\rfloor\right) \text {. }
$$

In this last equation, $\boldsymbol{S}_{p c}$ is estimated using the same decomposition of $\boldsymbol{c}_{j}$ as before (see Eq. (44)),

$$
\begin{array}{r}
\boldsymbol{S}_{p c}=\frac{1}{N_{s}} \sum_{j=1}^{N_{s}} \boldsymbol{p}_{j} \boldsymbol{\mu}_{c_{j}}^{H}+\frac{1}{N_{s}} \sum_{j=1}^{N_{s}} \boldsymbol{p}_{j} \boldsymbol{x}_{j}^{H} \\
\approx \boldsymbol{S}_{p p}\left\lceil\boldsymbol{\sigma}^{-2}\right\rfloor \boldsymbol{L}\lceil\boldsymbol{\alpha}\rfloor \boldsymbol{\Omega}_{c}^{H}
\end{array}
$$

\section{B.4 Sampling of $\gamma^{2}$}

Using the expression (40), the posterior for $\gamma^{2}$ can be written as follows:

$$
\begin{aligned}
{\left[\gamma^{2} \mid \infty\right] } & \propto \prod_{j=1}^{N_{s}}\left[\boldsymbol{c}_{j} \mid \gamma^{2}\right]\left[\gamma^{2}\right] \\
& \propto \prod_{j=1}^{N_{s}} \mathcal{N}_{\mathbb{C}}\left(0, \gamma^{2} \boldsymbol{I}_{\kappa}\right) \mathcal{I} \mathcal{G}\left(a_{\gamma}, b_{\gamma}\right)
\end{aligned}
$$

The use of the conjugacy of the inverse-gamma with the Gaussian directly gives the expression the posterior:

$$
\left[\gamma^{2} \mid \infty\right] \propto \mathcal{I G}\left(a_{\gamma}+\kappa N_{s}, b_{\gamma}+\operatorname{Trace}\left(\boldsymbol{S}_{c c}\right)\right)
$$




\section{B.5 Sampling of $\alpha$}

Still using (40), the $k^{\text {th }}$ element of the vector $\boldsymbol{\alpha}$ is sampled as follows:

$$
\begin{aligned}
{\left[\boldsymbol{\alpha}_{k} \mid \infty\right] } & \propto \prod_{j=1}^{N_{s}}\left[\boldsymbol{p}_{j} \mid \boldsymbol{\alpha}_{k}, \infty\right]\left[\boldsymbol{\alpha}_{k}\right] \quad k=1, \ldots, \kappa \\
& \propto \prod_{j=1}^{N_{s}} \mathcal{N}_{\mathbb{C}}\left(\boldsymbol{L}\lceil\boldsymbol{\alpha}\rfloor \boldsymbol{c}_{j},\left\lceil\boldsymbol{\sigma}^{2}\right\rfloor\right) \mathcal{E}\left(\boldsymbol{a}_{\alpha_{k}}\right) .
\end{aligned}
$$

This can be identify to a real Gaussian, truncated over a positive support:

$$
\left[\boldsymbol{\alpha}_{k} \mid \infty\right] \propto \mathcal{N}_{\mathbb{R}}\left(\boldsymbol{\mu}_{\alpha_{k}}, \boldsymbol{\sigma}_{\alpha_{k}}^{2}\right) \mathbf{1}_{\left\{\boldsymbol{\alpha}_{k} \leq 0\right\}},
$$

$$
\begin{aligned}
& \text { with } \quad \boldsymbol{\sigma}_{\alpha_{k}}^{2}=\left(2 \boldsymbol{L}_{k}^{H}\left\lceil\boldsymbol{\sigma}^{-2}\right\rfloor \boldsymbol{L}_{k} \boldsymbol{S}_{c c_{k k}}\right)^{-1} \\
& \text { and } \quad \boldsymbol{\mu}_{\alpha_{k}}=2 \boldsymbol{\sigma}_{\alpha_{k}}^{2}\left(\Re \left\{\boldsymbol { L } _ { k } ^ { H } \lceil \boldsymbol { \sigma } ^ { - 2 } \rfloor \left(\boldsymbol{S}_{p c_{k}}+\boldsymbol{\alpha}_{k} \boldsymbol{L}_{k} \boldsymbol{S}_{c c_{k k}}\right.\right.\right. \\
& \left.\left.\left.-\boldsymbol{L}\lceil\boldsymbol{\alpha}\rfloor \boldsymbol{S}_{c c_{k}}\right)\right\}-\boldsymbol{a}_{\alpha_{k}}\right)
\end{aligned}
$$

where the single subscript notation $\boldsymbol{A}_{k}$ indicates the $\mathrm{k}^{\text {th }}$ column of the matrix $\boldsymbol{A}$ and $\Re$ is the real part operator.

\section{B.6 Sampling of $\sigma^{2}$}

From the prior assigned to $\boldsymbol{\sigma}^{2}$ and the likelihood function,

$$
\begin{aligned}
{\left[\boldsymbol{\sigma}^{2} \mid \infty\right] } & \propto \prod_{j=1}^{N_{s}}\left[\boldsymbol{p}_{j} \mid \infty\right]\left[\boldsymbol{\sigma}_{n}^{2}\right] \\
& \propto \prod_{j=1}^{N_{s}} \mathcal{N}_{\mathbb{C}}\left(\boldsymbol{L}\lceil\boldsymbol{\alpha}\rfloor \boldsymbol{c}_{j},\left\lceil\boldsymbol{\sigma}^{2}\right\rfloor\right) \mathcal{N}_{\mathbb{C}}\left(\mathbf{0},\left\lceil\boldsymbol{\sigma}^{2}\right\rfloor\right)
\end{aligned}
$$

Again using the conjugacy of the inverse-gamma with the Gaussian, the expression of the posterior becomes

$$
\left[\boldsymbol{\sigma}_{m}^{2} \mid \infty\right] \propto \mathcal{I G}\left(\boldsymbol{a}_{\sigma_{m}}+N_{s}, \boldsymbol{b}_{\sigma_{m}}+\boldsymbol{T}_{m m}\right) \quad m=1, \ldots, M
$$

with $\quad \boldsymbol{T}=\frac{1}{N_{s}} \sum_{j=1}^{N_{s}}\left(\boldsymbol{p}_{j}-\boldsymbol{L}\lceil\boldsymbol{\alpha}\rfloor \boldsymbol{c}_{j}\right)\left(\boldsymbol{p}_{j}-\boldsymbol{L}\lceil\boldsymbol{\alpha}\rfloor \boldsymbol{c}_{j}\right)^{H}$

$$
=\boldsymbol{S}_{p p}+\boldsymbol{L} \boldsymbol{S}_{c c} \boldsymbol{L}^{H}-\boldsymbol{S}_{p c} \boldsymbol{L}^{H}-\boldsymbol{L} \boldsymbol{S}_{c p}
$$

Making use of the expression of $\boldsymbol{S}_{p c}$ given in Eq. (49) and $\boldsymbol{S}_{c c}$ is replaced by its expression given in Eq. (46).

$$
\begin{gathered}
\boldsymbol{T}=\left(\boldsymbol{I}_{M}-\boldsymbol{B}\right) \boldsymbol{S}_{p p}\left(\boldsymbol{I}_{M}-\boldsymbol{B}\right)+\boldsymbol{L}\lceil\boldsymbol{\alpha}\rfloor \boldsymbol{W}_{c}\lceil\boldsymbol{\alpha}\rfloor \boldsymbol{L}^{H} \\
\text { where } \quad \boldsymbol{B}=\boldsymbol{L}\lceil\boldsymbol{\alpha}\rfloor \boldsymbol{\Omega}_{c}\lceil\boldsymbol{\alpha}\rfloor \boldsymbol{L}^{H}\left\lceil\boldsymbol{\sigma}^{-2}\right\rfloor=\boldsymbol{B}^{H},
\end{gathered}
$$

By doing so, it is visible that the semi-positivity of $\boldsymbol{T}$ is preserved.

\section{References}

[1] R. O. Schmidt. Multiple emitter location and signal parameter estimation. In Proc. RADC Spectrum Estimation Workshop, pages 243-258, 1979.
[2] J. Y. Chung. Rejection of flow noise using a coherence function method. The Journal of the Acoustical Society of America, 62(2):388-395, 1977.

[3] W.S. Burdic. Underwater Acoustic System Analysis. Englewood Cliffs, NJ: Prentice-Hall, 1991.

[4] J. A. Cadzow. Signal enhancement-a composite property mapping algorithm. IEEE Transactions on Acoustics, Speech, and Signal Processing, 36(1):49-62, Jan 1988.

[5] P. Forster. Generalized rectification of cross spectral matrices for arrays of arbitrary geometry. IEEE Transactions on Signal Processing, 49(5):972978, May 2001.

[6] P. Forster and T. Asté. Rectification of cross spectral matrices for arrays of arbitrary geometry. In Acoustics, Speech, and Signal Processing, 1999. Proceedings., 1999 IEEE International Conference on, volume 5, pages 2829-2832 vol.5, 1999.

[7] Benjamin A. Fenech. Accurate aeroacoustic measurements in closed-section hard-walled wind tunnels. $\mathrm{PhD}$ thesis, University of Southampton, June 2009.

[8] J. Bulté. Acoustic array measurements in aerodynamic wind tunnels: A subspace approach for noise suppression. In 13th AIAA/CEAS Aeroacoustics Conference, Rome, Italy, 2007.

[9] Blandine Arguillat, Denis Ricot, Christophe Bailly, and Gilles Robert. Measured wavenumber: Frequency spectrum associated with acoustic and aerodynamic wall pressure fluctuations. The Journal of the Acoustical Society of America, 128(4):1647$1655,2010$.

[10] Philippe Druault, Abbas Hekmati, and Denis Ricot. Discrimination of acoustic and turbulent components from aeroacoustic wall pressure field. Journal of Sound and Vibration, 332(26):7257 - 7278, 2013.

[11] E Salze, Christophe Bailly, Olivier Marsden, Emmanuel Jondeau, and Daniel Juvé. An experimental characterization of wall pressure wavevectorfrequency spectra in the presence of pressure gradients. In 20th AIAA/CEAS Aeroacoustics Conference, Atlanta, GA, number 2909, 2014.

[12] K Ehrenfried, L. Koop, A. Henning, and K. Kaepernick. Effects of wind-tunnel noise on array measurements in closed test sections. In Proceedings of BeBeC-2006, Berlin, Germany, 2006. 
[13] L. Koop and K Ehrenfried. Microphone-array processing for wind-tunnel measurements with strong background noise. In 14th AAIA/CEAS Aeroacoustics Conference, Vancouver, British Columbia, Canada, number 2907, 2008.

[14] Daniel Blacodon. Spectral estimation method for noisy data using a noise reference. Applied Acoustics, 72(1):11 - 21, 2011.

[15] D. Blacodon. Array processing for noisy data: Application for open and closed wind tunnels. AIAA Journal, 49(1):55-66, January 2011.

[16] C. J. Bahr and W. C. Horne. Advanced background subtraction applied to aeroacoustic wind tunnel testing. In 21st AIAA/CEAS Aeroacoustics Conference, Dallas, TX, number 2909, 2015.

[17] S.M. Jaeger, W.C. Horne, and C.S. Allen. Effect of surface treatment on array microphone self-noise. In 6th AIAA/CEAS Aeroacoustics Conference, Lahaina, USA, 2000, number 1937, 2000.

[18] R. P. Dougherty. Directional acoustic attentuation of planar foam rubber windscreens for phased arrays. In Proceedings of BeBeC-2012, Berlin, Germany., number 05, 2012.

[19] Quentin Leclère and Christophe Picard. Acoustic beamforming through a thin plate using vibration measurements. The Journal of the Acoustical Society of America, 137(6):3385-3392, 2015.

[20] D. Lecoq, C. Pézerat, J.-H. Thomas, and W.P. Bi. Extraction of the acoustic component of a turbulent flow exciting a plate by inverting the vibration problem. Journal of Sound and Vibration, 333(12):2505 - 2519, 2014.

[21] Jacob Benesty, Jingdong Chen, and Yiteng Huang. Microphone array signal processing, volume 1. Springer Science \& Business Media, 2008.

[22] S. Boll. Suppression of acoustic noise in speech using spectral subtraction. IEEE Transactions on Acoustics, Speech, and Signal Processing, 27(2):113120, Apr 1979.

[23] S. H. Jensen, P. C. Hansen, S. D. Hansen, and J. A. Sorensen. Reduction of broad-band noise in speech by truncated QSVD. IEEE Transactions on Speech and Audio Processing, 3(6):439-448, Nov 1995.

[24] Christopher R. Lowis. In-duct measurement techniques for the characterisation of broadband aeroengine noise. $\mathrm{PhD}$ thesis, University of Southampton, December 2007.
[25] Pieter Sijtsma, Alice Dinsenmeyer, Jérôme Antoni, and Quentin Leclere. Beamforming and other methods for denoising microphone array data. In 25th AIAA/CEAS Aeroacoustics Conference, page 2653, 2019 .

[26] Jørgen Hald. Removal of incoherent noise from an averaged cross-spectral matrix. The Journal of the Acoustical Society of America, 142(2):846-854, 2017.

[27] Michael Grant and Stephen Boyd. CVX: Matlab software for disciplined convex programming, version 2.1. http://cvxr.com/cvx, March 2014.

[28] Michael Grant and Stephen Boyd. Graph implementations for nonsmooth convex programs. In V. Blondel, S. Boyd, and H. Kimura, editors, Recent Advances in Learning and Control, Lecture Notes in Control and Information Sciences, pages 95-110. Springer-Verlag Limited, 2008. http://stanford.edu/ boyd/graph_dcp.html.

[29] Reha H Tütüncü, Kim-Chuan Toh, and Michael J Todd. Solving semidefinite-quadratic-linear programs using sdpt3. Mathematical programming, 95(2):189-217, 2003.

[30] Robert P Dougherty. Cross spectral matrix diagonal optimization. In 6th Berlin beamforming conference, 2016.

[31] Quentin Leclere, Nicolas Totaro, Charles Pézerat, Fabien Chevillotte, and Pascal Souchotte. Extraction of the acoustic part of a turbulent boundary layer from wall pressure and vibration measurements. In INTER-NOISE and NOISE-CON Congress and Conference Proceedings, volume 251, pages 816-824. Institute of Noise Control Engineering, 2015.

[32] S. H. Yoon and P. Nelson. A method for the efficient construction of acoustic pressure crossspectral matrices. Technical Report 281, Institute of Sound and Vibration Research, 1998.

[33] Liang Yu, Jerome Antoni, and Quentin Leclère. Spectral matrix completion by cyclic projection and application to sound source reconstruction from non-synchronous measurements. Journal of Sound and Vibration, 372:31 - 49, 2016.

[34] John Wright, Arvind Ganesh, Shankar Rao, Yigang Peng, and Yi Ma. Robust principal component analysis: Exact recovery of corrupted low-rank matrices via convex optimization. In Advances in neural information processing systems, pages 2080-2088, 2009. 
[35] A. Finez, A. Pereira, and Q. Leclère. Broadband mode decomposition of ducted fan noise using crossspectral matrix denoising. In Proceedings of Fan Noise 2015, Lyon, France, 2015.

[36] Sylvain Amailland, Jean-Hugh Thomas, Charles Pézerat, and Romuald Boucheron. Boundary layer noise subtraction in hydrodynamic tunnel using robust principal component analysis. The Journal of the Acoustical Society of America, 143(4):21522163, 2018.

[37] Andrews Sobral, Thierry Bouwmans, and El-hadi Zahzah. Lrslibrary: Low-rank and sparse tools for background modeling and subtraction in videos. In Robust Low-Rank and Sparse Matrix Decomposition: Applications in Image and Video Processing. CRC Press, Taylor and Francis Group., 2015.

[38] https://github.com/andrewssobral/ lrslibrary/. Accessed: May 8, 2020.

[39] Jørgen Hald. Denoising of cross-spectral matrices using canonical coherence. The Journal of the Acoustical Society of America, 146(1):399-408, 2019.

[40] Christopher M Bishop. Pattern recognition and machine learning. Springer, 2006.

[41] Dani Gamerman and Hedibert F Lopes. Markov chain Monte Carlo: stochastic simulation for Bayesian inference. Chapman and Hall/CRC, 2006.

[42] Andrew Gelman, John B Carlin, Hal S Stern, David B Dunson, Aki Vehtari, and Donald B Rubin. Bayesian data analysis. Chapman and Hall/CRC, 2014.

[43] Jérôme Antoni, Charles Vanwynsberghe, Thibaut Le Magueresse, Simon Bouley, and Laurent Gilquin. Mapping uncertainties involved in sound source reconstruction with a cross-spectral-matrix-based Gibbs sampler. The Journal of the Acoustical Society of America, 146(6):4947-4961, 2019.

[44] Torbjørn Eltoft, Taesu Kim, and Te-Won Lee. On the multivariate laplace distribution. IEEE Signal Processing Letters, 13(5):300-303, 2006.

[45] Ennes Sarradj, Gert Herold, Pieter Sijtsma, Roberto Merino-Martinez, Anwar Malgoezar, Mirjam Snellen, Thomas Geyer, Christopher Bahr, Ric Porteous, Danielle Moreau, and Con Doolan. A microphone array method benchmarking exercise using synthesized input data. In 23rd AIAA/CEAS Aeroacoustics Conference, June 2017.
[46] Alice Dinsenmeyer, Jérôme Antoni, Quentin Leclere, and Antonio Pereira. On the denoising of cross-spectral matrices for (aero) acoustic applications. In 7th Berlin beamforming conference, 2018.

[47] G. M. Corcos. Resolution of pressure in turbulence. The Journal of the Acoustical Society of America, 35(2), 1963.

[48] Alice Dinsenmeyer, Quentin Leclere, Jérôme Antoni, and Emmanuel Julliard. Comparison of microphone array denoising techniques and application to flight test measurements. In 25th AIAA/CEAS Aeroacoustics Conference, page 2744, 2019.

[49] Peter Ahrendt. The multivariate gaussian probability distribution. Technical University of Denmark, Tech. Rep, 2005.

[50] Kaare Brandt Petersen and Michael Syskind Pedersen. The matrix cookbook (version: November 15, 2012), 2012. 